\title{
Modulated Branching Processes, Origins of Power Laws and Queueing Duality
}

\author{
Predrag R. Jelenković Jian Tan \\ Department of Electrical Engineering \\ Columbia University, New York, NY 10027 \\ \{predrag, jiantan\}@ee.columbia.edu
}

September 2007; revised July 2008

\begin{abstract}
Power law distributions have been repeatedly observed in a wide variety of socioeconomic, biological and technological areas. In many of the observations, e.g., city populations and sizes of living organisms, the objects of interest evolve due to the replication of their many independent components, e.g., births-deaths of individuals and replications of cells. Furthermore, the rates of the replication are often controlled by exogenous parameters causing periods of expansion and contraction, e.g., baby booms and busts, economic booms and recessions, etc. In addition, the sizes of these objects often have reflective lower boundaries, e.g., cities do not fall bellow a certain size, low income individuals are subsidized by the government, companies are protected by bankruptcy laws, etc.

Hence, it is natural to propose reflected modulated branching processes as generic models for many of the preceding observations. Indeed, our main results show that the proposed mathematical models result in power law distributions under quite general polynomial Gärtner-Ellis conditions, the generality of which could explain the ubiquitous nature of power law distributions. In addition, on a logarithmic scale, we establish an asymptotic equivalence between the reflected branching processes and the corresponding multiplicative ones. The latter, as recognized by Goldie (1991) [32, is known to be dual to queueing/additive processes. We emphasize this duality further in the generality of stationary and ergodic processes.
\end{abstract}

Keywords: Modulated branching processes, reflective/absorbing barriers, reflected multiplicative processes, proportional growth models, power law distributions, heavy tails, subexponential distributions, queueing processes, reflected additive random walks, Cramér large deviations, polynomial Gärtner-Ellis conditions.

The preliminary version of this work has appeared in the extended abstract in Proceedings of the Forty-Fourth Annual Allerton Conference, Allerton House, UIUC, Illinois, USA, September 2006. Technical Report EE2007-09-25, Department of Electrical Engineering, Columbia University, New York, NY, September 25, 2007. 


\section{Introduction}

Power law distributions are found in a wide range of domains, ranging from socioeconomic to biological and technological areas. Specifically, these types of distributions describe the city populations, species-area relationships, sizes of living organisms, values of companies, distributions of wealth, and more recently, sizes of documents on the Web, visitor access patterns on Web sites, etc. Hence, one would expect that there exist universal mathematical laws that explain this ubiquitous nature of power law distributions. To this end, we propose a class of models, termed modulated branching processes with reflective lower barriers that, under quite general polynomial Gärtner-Ellis conditions, result in power law distributions.

Empirical observations of power laws have a long history, starting from the discovery by Pareto [60] in 1897 that a plot of the logarithm of the number of incomes above a level against the logarithm of that level yields points close to a straight line, which is essentially equivalent to saying that the income distribution follows a power law. Hence, power law distributions are often called Pareto distributions; for more recent study on income distributions see 16, 55, 21, 64, 65. In a different context, early work by Arrhenius [4] in 1921 conjectured a power law relationship between the number of species and the census area, which was followed by Preston's prediction in 63 that the slope on the $\log / \log$ species-area plot has a canonical value equal to 0.262; for additional information and measurements on species-area relationships see [18, 62, 46]. Interestingly, there also exists a power law relationship between the rank of the cities and the population of the corresponding cities. This was proposed by Auerbach [8] in 1913 and later studied by Zipf [73] in 1949, after whom power law is also known as Zipf's law. Ever since, much attention on both empirical examinations and explanations of the city size distributions have been drawn [73, 37, 28, 68, 61, 2]. Similar observations have been made for firm sizes [3], language family sizes [72], and even the gene family and protein statistics [36, 69, 54, 13. It is maybe even more surprising that many features of the Internet are governed by power laws, including the distribution of pages per Web site [34, the page request distribution [20, 12, the file size distribution [25, 40], Ethernet LAN traffic [49], World Wide Web traffic [19], the number of visitors per Web site [35, 1], the distribution of scenes in MPEG video streams [39] and the distribution of the indegrees and outdegrees in the Web graph as well as the physical network connectivity graph [26, 9, 48, 56]. In socio-economic areas, in addition to income distributions, the fluctuations in stock prices have also been observed to be characterized by power laws $[29,50$. This paragraph only exemplifies various observations of power laws; for a more complete survey see [57].

Hence, these repeated empirical observations of power laws over a period of more than a hundred years strongly suggest that there exist general mathematical laws that govern these phenomena. In this regard, after carefully examining the situations that result in power laws, we discover that most of them are characterized by the following three features. First, in the vast majority of these observations, e.g., city populations and sizes of living organisms, the objects of interest evolve due to the replication of their many independent components, e.g., birth-deaths of individuals and replications of cells. Secondly, the rate of replication of the many components is often controlled by exogenous parameters causing periods of baby booms and busts, economic growths and recessions, etc. Thirdly, the sizes of these objects often have lower boundaries, e.g., cities do not fall bellow a certain size, low income individuals are subsidized by the government, companies are protected by bankruptcy laws, etc.

In order to capture the preceding features, it is natural to propose modulated branching processes (MBP) with reflective or absorbing barriers as generic models for many of the observations of power laws. Indeed, one of our main results, presented in Theorem 3 , shows that MBPs with reflective barriers almost invariably produce power law distributions under quite 
general polynomial Gärtner-Ellis conditions. The generality of our results could explain the ubiquitous nature of power law distributions. Furthermore, an informal interpretation of our main results, stated in Theorems 3 and 4 of Section 3. suggests that alternating periods of expansions and contractions, e.g., economic booms and recessions, are primarily responsible for the appearance of power law distributions. Actually, Theorem 4 shows that the distribution of the reflected MBP is exponentially bounded if the process always contracts. From a mathematical perspective, we develop a novel sample path technique for analyzing reflected modulated branching processes since these objects appear new and the traditional methods for investigating branching processes [7] do not directly apply; a preliminary version of this work has appeared in the extended abstract in [42].

Formal description of our reflected modulated branching process (RMBP) model is given in Section 2, In the singular case when the number of individuals born in each state of the modulating process is constant, our model reduces to a reflected multiplicative process. A rigorous connection (duality) between the reflected multiplicative processes (RMPs) and queueing theory was established in Section 5 of Goldie (1991) [32]; this duality was repeatedly observed and used later in, e.g., [71, 33. In Subsection 2.1 we further emphasize this duality in the context of stationary and ergodic processes. We would like to point out that this duality makes a vast literature on queueing theory directly applicable to the analysis of RMPs. As a direct consequence of this connection, in Subsection 2.1 we translate several well known queueing results to the context of RMPs. Informally, these results show that the role which exponential distributions play in queueing theory, and in additive reflected random walks in general, is represented by power low distributions in the framework of RMPs/RMBPs. Furthermore, this relationship appears to reduce the debate on the relative importance of power law versus exponential distributions/models to the analogous question of the prevalence of proportional growth versus additive phenomena. Interestingly, the power law distribution satisfies the memoryless property in the multiplicative world, playing an equivalent role to the memoryless exponential distribution in the additive world. Indeed, if $\mathbb{P}[M>x]=x^{-\alpha}, \alpha>$ $0, x \geq 1$, then, for $x, y \geq 1$, we obtain $\mathbb{P}[M>x y \mid M>x]=\mathbb{P}[M>y]$.

Furthermore, this duality immediately implies and generalizes many of the prior results in the area of RMPs and power laws, e.g., see [51, 52, 50, 71]. Furthermore, we would like to point out that the reflective nature of the barrier, assumed in the previous studies, is not essential for producing power law distributions. Indeed, one only needs a positive lower barrier, e.g., porous, absorbing or reflective one, which is a natural condition since no physical object or socioeconomic one can approach zero arbitrarily close without repelling from it or simply disappearing. In many areas, objects of interest may not have a strictly reflecting barrier, but rather a porous one, e.g., cities may degenerate, bankruptcy protection may sometimes fail and a company can be liquidated. In these cases, the power law effect follows from the well-known queueing results on cycle maximum that we briefly stated in Subsection 2.2. This observation presents a rigorous explanation for the previous study in 11] that argued heuristically how multiplicative processes with absorbing barriers can result in power laws.

Here, we would like to point out that the stochastic difference equation $\left(M_{n+1}=J_{n} M_{n}+\right.$ $\left.Q_{n}\right)$ with random coefficients is closely related to RMPs and is known to produce power law distributions. It appears that the first rigorous study of this process was done by Kesten in 1973 [47; for later investigations of this model see [22, 23] and the references therein. In addition, we refer the reader to equations $(1.1)-(1.6)$ on p.126 of [32] for other related stochastic recursions of multiplicative nature that produce power laws.

Next, it is easy to see that RMBPs reduce to RMPs in the special case when constant number of individuals are born in each state of the modulating process. However, our main result, Theorem 3, reveals a general asymptotic equivalence between the power law exponent 
of a RMBP and the corresponding RMP. In other words, Theorem 3 discovers the asymptotic insensitivity of the power law exponent on the conditional distributions of the reflected branching process beyond their conditional mean values. Furthermore, for the special case when the modulating process is i.i.d., we sharpen the result on the logarithmic asymptotics of Theorem [3 to the exact one in Theorem 7 by using the implicit renewal theory of Goldie 1991 [32].

In some domains, e.g., the growth of living organisms, the objects always grow (basically never shrink) up until a certain random time. Huberman and Adamic 34] also propose this model as an explanation of the growth dynamics of the World Wide Web by arguing that the observation time is an exponential random variable. This notion has been revisited in [64] and generalized to a larger family of random processes observed at an exponential random time [66]. In this regard, in Subsection 5.1.2, we study randomly stopped modulated branching processes and show, under more general conditions than the preceding studies, that the resulting variables follow power laws.

In regard to the previously mentioned situations with absorbing barriers, we discuss MBP with an absorbing barrier in Subsection 5.2 and argue that it leads to power law distributions as well. We conjecture that these types of models can be natural candidates for describing the bursts of requests at popular Internet Web sites, often referred to as hotspots.

Based on our new model, we discuss two related phenomena: truncated power laws and double Pareto distributions. We argue that one can obtain a truncated power law distribution by adding an upper barrier to RMBP, similarly as the truncated geometric distributions appear in queueing theory, e.g., finite buffer $M / M / 1$ queue. Furthermore, by the duality of RMBP and queueing theory, we give two new natural explanations of the origins of double Pareto distributions that have been observed in practice. In the queueing context, it has been shown that the tail of the queue length distribution exhibits different decay rates in the heavy-traffic and large deviation regime, respectively [59]; similar behavior of the queue length distribution was attributed to the multiple time scale arrivals in [38]. We claim that the preceding two mechanisms, when translated to the proportional growth context, provide natural explanations of the double Pareto distributions.

Finally, we would like to mention that there might be other non multiplicative mechanisms that result in power law distributions, e.g., the randomly typing model used to explain the power law distribution of frequencies of words in natural languages [57] and the highly optimized tolerance studied in [15]. Very recently, the new power law phenomenon in the situations where jobs have to restart from the beginning after a failure was discovered in [27] and further studied in [70, 6]; equivalently in the communication context, the retransmission based protocols in data networks were shown to almost invariably lead to power laws and, in general, heavy tails in [41, 44, 43, 45]. For a recent survey on various mechanisms that result in power laws see [57].

The rest of the paper is organized as follows. After introducing the modulated branching processes in Section 2, we study the duality between the queueing theory and the multiplicative processes with reflected barriers in Subsection 2.1 and absorbing barriers in Subsection 2.2. respectively. Then, we present our main results in Section 3 on the logarithmic asymptotics of the stationary distribution of the reflected modulated branching process and the corresponding multiplicative one, which is followed by the study of the exact asymptotics under the more restrictive conditions in Section 4. As further extensions, we discuss three related models in Section 5, i.e., randomly stopped processes in Subsection 5.1 modulated branching processes with absorbing barriers in Subsection 5.2 and truncated power laws in Subsection 5.3. In the end, Section 6 presents the majority of the technical proofs that have been deferred from the preceding sections for increased readability. 


\section{Reflected Modulated Branching Processes}

In this section we formally describe our model. Let $\left\{J_{n}\right\}_{n>-\infty}$ be a stationary and ergodic modulating process that takes values in positive integers. Define a family of independent, nonnegative, integer-valued random variables $\left\{B_{n}^{i}(j)\right\},-\infty<i, j, n<\infty$, which are independent of the modulating process $\left\{J_{n}\right\}$. In addition, for fixed $j$, variables $\left\{B(j), B_{n}^{i}(j)\right\}$ are identically distributed with $\mu(j) \triangleq \mathbb{E}[B(j)]<\infty$.

Definition 1. A Modulated Branching Process (MBP) $\left\{Z_{n}\right\}_{n=0}^{\infty}$ is recursively defined by

$$
Z_{n+1} \triangleq \sum_{i=1}^{Z_{n}} B_{n}^{i}\left(J_{n}\right)
$$

where the initial value $Z_{0}$ is a positive integer. For increased clarity, we may explicitly write $\left\{Z_{n}^{l}\right\}$ when $Z_{0}=l$.

Definition 2. For any $l \in \mathbb{N}$ and an integer valued $\Lambda_{0}$, a Reflected Modulated Branching Process (RMBP) $\left\{\Lambda_{n}\right\}_{n=0}^{\infty}$ is recursively defined as

$$
\Lambda_{n+1} \triangleq \max \left(\sum_{i=1}^{\Lambda_{n}} B_{n}^{i}\left(J_{n}\right), l\right) .
$$

Remark 1. These types of modulated branching processes with a reflecting barrier appear to be new and, thus, the traditional methods for the analysis of branching processes [7] do not seem to directly apply.

Remark 2. A more general framework would be to define

$$
Z_{n+1}=\int_{0}^{Z_{n}} B_{n}^{t}\left(J_{n}(t)\right) d \nu(t)
$$

for any real measure $\nu$ and, similarly,

$$
\Lambda_{n+1}=\max \left(\int_{0}^{\Lambda_{n}} B_{n}^{t}\left(J_{n}(t)\right) d \nu(t), l\right),
$$

where $l>0$ and $B_{n}^{t}\left(J_{n}(t)\right)$ is $\nu$-measurable. We refrain from this generalization since it introduces additional technical difficulties without much new insight.

Now, we present the basic limiting results on the convergence to stationarity of $Z_{n}$ and $\Lambda_{n}$.

Lemma 1. If $\mathbb{E} \log \mu\left(J_{0}\right)<0$, then a.s., we have

$$
\lim _{n \rightarrow \infty} Z_{n}=0 .
$$

Proof. For all $n \geq 1$, let $W_{n}=Z_{n} / \Pi_{n-1}^{0}$, where $\Pi_{n}^{0}=\prod_{i=0}^{n} \mu\left(J_{i}\right)$. It is easy to check that $W_{n}$ is a positive martingale with respect to the filtration $\mathcal{F}_{n}=\sigma\left(J_{i}, Z_{i}, 0 \leq i \leq n-1\right)$. Hence, by the martingale convergence theorem (see Theorem 35.5. of [10]), almost surely (a.s.) as $n \rightarrow \infty$,

$$
W_{n} \rightarrow W<\infty
$$

Next, since $\left\{J_{n}\right\}$ is stationary and ergodic, so is $\left\{\mu\left(J_{n}\right)\right\}$, and therefore, a.s.,

$$
\frac{\log \Pi_{n-1}^{0}}{n}=\frac{1}{n} \sum_{i=0}^{n-1} \log \mu\left(J_{i}\right) \rightarrow \mathbb{E} \log \mu\left(J_{0}\right)<0 \quad \text { as } n \rightarrow \infty .
$$

Thus, $\Pi_{n-1}^{0} \rightarrow 0$ as $n \rightarrow \infty$, which, by recalling (15) and $Z_{n}=W_{n} \Pi_{n-1}^{0}$, finishes the proof. 
Next, let $Z_{-n}$ be the number of individuals at time 0 in an unrestricted branching process that starts at time $-n$ with $l$ individuals; when needed for clarity, we will use the notation $Z_{-n}^{l}$ to explicitly indicate the initial state $l$.

Lemma 2. Assume $\mathbb{E} \log \mu\left(J_{0}\right)<0$, then, for any a.s. finite initial condition $\Lambda_{0}, \Lambda_{n}$ converges in distribution to

$$
\Lambda \stackrel{d}{=} \max _{n \geq 0} Z_{-n}
$$

Proof. First, assume that $\Lambda_{0}=l$ and let $Z_{n}^{k}$ be the number of individuals at time $n$ in an unrestricted branching process that starts at time $k$ with $l$ individuals. Then, by stationarity of $\left\{J_{n}\right\}$, we have $Z_{n}^{k} \stackrel{d}{=} Z_{k-n}$. Clearly,

$$
\Lambda_{1}=\max \left(\sum_{i=1}^{l} B_{1}^{i}\left(J_{1}\right), l\right) \stackrel{d}{=} \max \left\{Z_{-1}, Z_{0}\right\},
$$

and, by induction and stationarity, it is easy to show

$$
\Lambda_{n} \stackrel{d}{=} \max \left(Z_{-n}, Z_{-(n-1)}, \cdots, Z_{-1}, Z_{0}\right)
$$

where $Z_{-n}, \cdots, Z_{0}$ are defined on the same sequence $\left\{B_{k}^{i}\left(J_{k}\right)\right\}_{i \geq 1},-n \leq k \leq 0$. Hence, by monotonicity, we obtain

$$
\mathbb{P}\left[\Lambda_{n}>x\right] \rightarrow \mathbb{P}[\Lambda>x] \text { as } n \rightarrow \infty .
$$

Now, if $\Lambda_{n}^{\Lambda_{0}}$ is a process defined on the same sequence $\left\{B_{n}^{i}\left(J_{n}\right)\right\}$ with the initial condition $\Lambda_{0} \geq l$, then, it is easy to see that

$$
\Lambda_{n}^{\Lambda_{0}} \geq \Lambda_{n} \geq l, \text { for all } n
$$

implying

$$
\mathbb{P}\left[\Lambda_{n}^{\Lambda_{0}}>x\right] \geq \mathbb{P}\left[\Lambda_{n}>x\right] .
$$

Next, if we define the stopping time $\tau$ to be the first time when $\Lambda_{n}^{\Lambda_{0}}$ hits the boundary $l$, then the preceding monotonicity implies that $\Lambda_{n}=\Lambda_{n}^{\Lambda_{0}}$ for all $n \geq \tau$. Using this observation, we obtain

$$
\begin{aligned}
\mathbb{P}\left[\Lambda_{n}^{\Lambda_{0}}>x\right] & =\mathbb{P}\left[\Lambda_{n}^{\Lambda_{0}}>x, \tau>n\right]+\mathbb{P}\left[\Lambda_{n}^{\Lambda_{0}}>x, \tau \leq n\right] \\
& =\mathbb{P}\left[\Lambda_{n}^{\Lambda_{0}}>x, \tau>n\right]+\mathbb{P}\left[\Lambda_{n}>x, \tau \leq n\right] \\
& \leq \mathbb{P}[\tau>n]+\mathbb{P}\left[\Lambda_{n}>x\right] .
\end{aligned}
$$

Next, by Lemma 1, $\tau$ is a.s. finite and, thus, by (7) and (8), we conclude

$$
\lim _{n \rightarrow \infty} \mathbb{P}\left[\Lambda_{n}>x\right]=\lim _{n \rightarrow \infty} \mathbb{P}\left[\Lambda_{n}^{\Lambda_{0}}>x\right]=\mathbb{P}[\Lambda>x] .
$$

\subsection{Reflected Multiplicative Processes and Queueing Duality}

Note that in the special case $B_{n}^{i}\left(J_{n}\right) \equiv J_{n}$, reflected modulated branching processes reduce to reflected multiplicative processes with $J_{n}$ being integer valued. In general, using the definition in (3), $J_{n}$ can be relaxed to take any positive real values. Hence, in this subsection we assume that $\left\{J_{n}\right\}_{n \geq 0}$ is a positive, real valued process. 
Definition 3. For $l>0$ and $M_{0}<\infty$, define a Reflected Multiplicative Process (RMP) as

$$
M_{n+1}=\max \left(M_{n} \cdot J_{n}, l\right), \quad n \geq 0 .
$$

The preceding RMP model was studied by Goldie in 1991 [32]; for later considerations of this model see [71, 52, 51, 28, 33, 25]. Goldie [32] also shows a direct connection (duality) between RMP and queuing theory in Section 5 of [32] for the case when $\left\{J_{n}\right\}$ is an i.i.d. sequence. Here, we study this duality further in the generality of stationary and ergodic processes.

Without loss of generality we can assume $l=1$, since we can always divide (9) by $l$ and define $M_{n}^{1}=M_{n} / l$. Now, let $X_{n}=\log J_{n}$ and $Q_{n}=\log M_{n}$ with the standard conventions $\log 0=-\infty$ and $e^{-\infty}=0$. Then, for $l=1$, equation (9) is equivalent to

$$
Q_{n+1}=\max \left(Q_{n}+X_{n}, 0\right),
$$

which is the workload (waiting-time) recursion in a single server (FIFO) queue.

Lemma 3. If $\mathbb{E} \log J_{n}<0$, then $M_{n}$ converges in distribution to an a.s. finite random variable $M$ that satisfies

$$
M \stackrel{d}{=} \sup _{n \geq 0} \Pi_{n}
$$

where $\Pi_{0}=1, \Pi_{n}=\prod_{i=-n}^{-1} J_{i}, n \geq 1$.

Proof. By the classical result of Loynes [53], $Q_{n}$, defined by (10), converges in distribution to an a.s. finite stationary limit $Q$ if $\mathbb{E} X_{n}=\mathbb{E} \log J_{n}<0$ and, furthermore,

$$
Q \stackrel{d}{=} \sup _{n \geq 0} S_{n}
$$

where $S_{0}=0$ and $S_{n}=\sum_{i=-n}^{-1} X_{i}$. This implies the convergence in distribution of $M_{n}$ to

$$
M \stackrel{d}{=} e^{\sup _{n \geq 0} S_{n}}=\sup _{n \geq 0} e^{S_{n}}=\sup _{n \geq 0} \Pi_{n} .
$$

The following theorem is a direct corollary of Theorem 1 in [31]; see also Theorem 3.8 in [17] and, for a more recent presentation, we refer the reader to [30].

Theorem 1. Let $\left\{J_{n}\right\}_{n \geq 1}$ be a stationary and ergodic sequence of positive random variables. If there exists a function $\Psi$ and positive constants $\alpha^{*}$ and $\varepsilon^{*}$ such that

1) $n^{-1} \log \mathbb{E}\left[\left(\Pi_{n}\right)^{\alpha}\right] \rightarrow \Psi(\alpha)$ as $n \rightarrow \infty$ for $\left|\alpha-\alpha^{*}\right|<\varepsilon^{*}$,

2) $\Psi$ is finite and differentiable in a neighborhood of $\alpha^{*}$ with $\Psi\left(\alpha^{*}\right)=0, \Psi^{\prime}\left(\alpha^{*}\right)>0$, and

3) $\mathbb{E}\left[\left(\Pi_{n}\right)^{\alpha^{*}+\varepsilon}\right]<\infty$, for $n \geq 1$ and some $\varepsilon>0$,

then

$$
\lim _{x \rightarrow \infty} \frac{\log \mathbb{P}[M>x]}{\log x}=-\alpha^{*}
$$


Remark 3. We refer to conditions 1) - 3) as the polynomial Gärtner-Ellis conditions. Note that condition 2) can be relaxed such that $\Psi$ is only differentiable at $\alpha^{*}$ and condition 3) can be weakened to $\varepsilon=0$ [31. Since conditions 2) and 3) are used for Theorem 3 in Section 3, we keep the current form to provide a unified framework. Also, it is worth noting that the multiplicative process $\Pi_{n}$ without the reflective boundary would essentially follow the lognormal distribution, as it was recently observed in [33] (this is similar to the fact that the unrestricted additive random walk is approximated well by Normal distribution). However, we would like to reemphasize that the lower boundary $l$ is not just a mathematical artifact, but a very natural condition since no physical object can approach zero arbitrarily close without either repelling (reflecting) from it or vanishing (absorbing); the absorbing boundary will be discussed in the following Subsection 2.2 .

Here, we illustrate the preceding theorem by the following examples. Assume that $\left\{A_{n}\right\},\left\{C_{n}\right\}$ are two mutually independent sequences, and let $J_{n}=e^{A_{n}-C_{n}}$. Then the quantity $Q_{n} \triangleq$ $\log M_{n}$, where $M_{n}$ is defined in (9), satisfies

$$
Q_{n+1}=\left(Q_{n}+A_{n}-C_{n}\right)^{+} .
$$

The first two examples assume that $\left\{A_{n}\right\},\left\{C_{n}\right\}$ are two i.i.d. sequences, the third example takes $\left\{J_{n}\right\}$ to be a Markov chain, and in the last example, $\left\{J_{n}\right\}$ is modulated by a Markov chain $\left\{X_{n}\right\}$.

Example 1. If $\left\{A_{n}\right\},\left\{C_{n}\right\}$ follow exponential distributions, $\mathbb{P}\left[C_{n}>x\right]=e^{-\mu x}, \mathbb{P}\left[A_{n}>x\right]=$ $e^{-\lambda x}$ and $\lambda<\mu$, then $Q_{n}$ represents the waiting time in a $M / M / 1$ queue. By Theorem 9.1 of [5], the stationary waiting time in a $M / M / 1$ queue is distributed as

$$
\mathbb{P}[Q>x]=\frac{\lambda}{\mu} e^{-(\mu-\lambda) x}, \quad x \geq 0,
$$

which equivalently yields a power law distribution for $M$,

$$
\mathbb{P}[M>x]=\mathbb{P}[Q>\log x]=\frac{\lambda}{\mu x^{\mu-\lambda}}, \quad x \geq 1
$$

with power exponent $\alpha=\mu-\lambda$.

Example 2. If $\left\{A_{n}\right\},\left\{C_{n}\right\}$ are two i.i.d Bernoulli processes with $\mathbb{P}\left[A_{n}=1\right]=1-\mathbb{P}\left[A_{n}=\right.$ $0]=p, \mathbb{P}\left[C_{n}=1\right]=1-\mathbb{P}\left[C_{n}=0\right]=q, p<q$. Then, the elementary queueing/Markov chain theory shows that the stationary distribution of $Q_{n}$, as defined in (13), is geometric $\mathbb{P}[Q \geq j]=(1-\rho) \rho^{j}, j \geq 0$, where $\rho=p(1-q) / q(1-p)<1$. Therefore,

$$
\mathbb{P}[M \geq x]=\mathbb{P}[Q \geq \log x]=\rho^{\lfloor\log x\rfloor}, \quad x \geq 1 .
$$

Since $\log x-1<\lfloor\log x\rfloor \leq \log x$, it is easy to conclude that

$$
\frac{1}{x^{\log (1 / \rho)}} \leq \mathbb{P}[M \geq x]<\frac{1}{\rho x^{\log (1 / \rho)}} .
$$

Example 3. If $\left\{J_{n}\right\}$ is a Markov chain taking values in a finite set $\Sigma$ and possessing an irreducible transition matrix $Q=(q(i, j))_{i, j \in \Sigma}$, then the function $\Psi$ defined in Theorem 1 can be explicitly computed. To this end, define matrix $Q_{\alpha}$ with elements

$$
q_{\alpha}(i, j)=q(i, j) j^{\alpha}, \quad i, j \in \Sigma \text {. }
$$


By Theorem 3.1.2 of [24], we have as $n \rightarrow \infty$,

$$
n^{-1} \log \mathbb{E}\left[\left(\Pi_{n}\right)^{\alpha}\right] \rightarrow \log \left(\operatorname{dev}\left(Q_{\alpha}\right)\right),
$$

where $\operatorname{dev}\left(Q_{\alpha}\right)$ is the Perron-Frobenius eigenvalue of matrix $Q_{\alpha}$. To illustrate this result, we take $\Sigma=\{u, d\}$ where $u=1 / d>1$, and $q(d, u)=q, q(d, d)=1-q, q(u, d)=p, q(u, u)=1-p$ where $p>q$. It is easy to compute

$$
Q_{\alpha}=\left(\begin{array}{cc}
(1-p) u^{\alpha} & p d^{\alpha} \\
q u^{\alpha} & (1-q) d^{\alpha}
\end{array}\right)
$$

and, by letting $\log \left(\operatorname{dev}\left(Q_{\alpha}\right)\right)=0$, we obtain

$$
\alpha^{*}=\frac{\log (1-q)-\log (1-p)}{\log u} .
$$

Example 4 (double Pareto). If $\left\{J_{n} \equiv J\left(X_{n}\right\}\right.$ is modulated by a Markov chain $X_{n}$, we argue that $\mathbb{P}[M>x]$ can have different asymptotic decay rates over multiple time scales. This phenomenon was investigated in 38 , in the queueing context and formulated as Theorem 3 therein. To visualize this phenomena, we study the following example. Consider a Markov process $X_{n}$ of two states (say $\{1,2\}$ ) with transition probabilities $p_{12}=1 / 5000, p_{21}=1 / 10$, and $\mathbb{P}[J(1)=1.2]=1-\mathbb{P}[J(1)=0.6]=0.5, \mathbb{P}[J(2)=1.7]=1-\mathbb{P}[J(2)=0.25]=0.6$. The corresponding simulation result for $5 \times 10^{7}$ trials is presented in Figure 1, We observe from this figure a double Pareto distribution for $M$, which provides a new explanation to the origins of double Pareto distributions as compared to the one in [67].

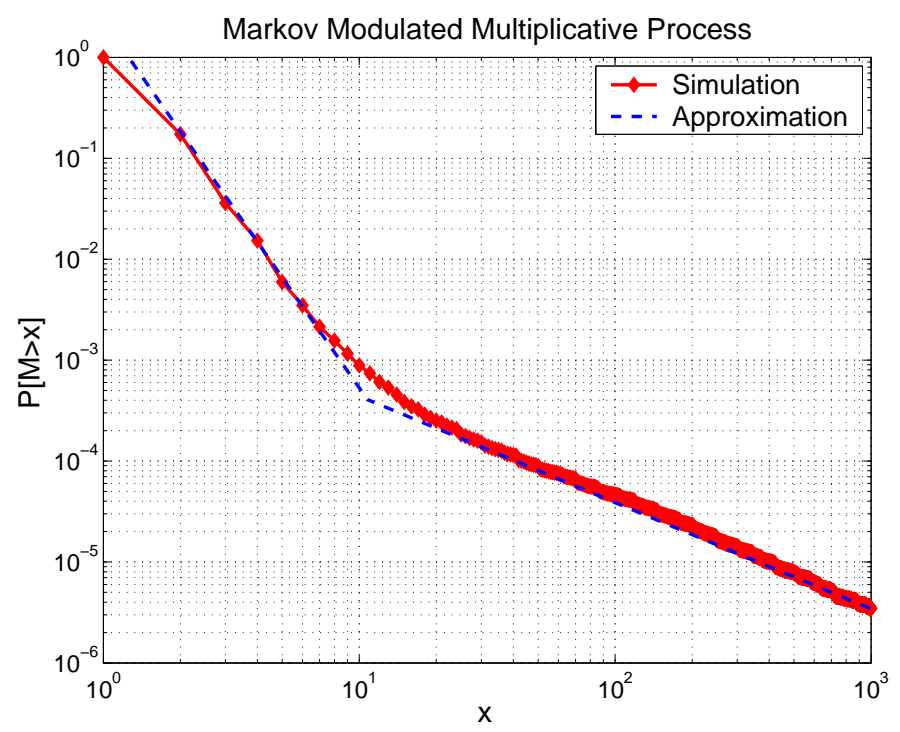

Figure 1: Illustration for Example 4 of the double Pareto distribution.

Remark 4. For reasons of simplicity, we have chosen $\left\{J_{n}\right\}$ in all of the preceding examples to be Markovian. However, Theorem 1 extends beyond the Markovian framework, e.g., $\left\{J_{n}\right\}$ can be a semi-Markov process where the periods of (sojourn) time that the process spends in a state are asymptotically exponential but not necessarily memoryless. 


\subsection{Multiplicative Processes with Absorbing Barriers and Cycle Maximum}

As briefly discussed in the introduction, we explained that the reflective nature of the barrier is not essential for producing power law distributions. Indeed, one only needs a positive lower barrier, e.g., porous, absorbing or reflective one, which is a natural condition since no physical objects or socioeconomic ones can approach zero arbitrarily close without repelling from it or simply disappearing. To illustrate the situations when the objects can vanish, we name a few examples, e.g., cities may degenerate, bankruptcy protection may sometimes fail and a company can be liquidated. In these cases, the power law effect follows from the well-known queueing result on cycle maximum that is stated in Theorem 2 below. We also discuss in Subsection 5.2 a more complicated situation when newly generated objects in the system can arrive/appear or leave/disappear.

Following the notation from Chapter VIII of [5], for a sequence of positive i.i.d. random variables $\left\{J, J_{n}\right\}_{n \geq 1}$, denote by $G_{+}$the ladder height distribution of the random walk $\left\{S_{n}=\right.$ $\left.\sum_{i=1}^{n} \log J_{i}\right\}_{n \geq 1}$ with $\left\|G_{+}\right\|=\mathbb{P}\left[S_{n} \leq 0\right.$ for all $\left.n \geq 1\right]$, and define the stopping time $\tau \triangleq \inf \{n$ : $\left.S_{n} \leq 0, n \geq 1\right\}$ with the corresponding cycle maximum $M_{\tau} \triangleq \sup \left\{\prod_{i=1}^{n} J_{i}: 1 \leq n \leq \tau\right\}$; here we assume, without loss of generality, that the absorbing barrier is equal to 1 .

Theorem 2. If the sequence $\left\{\log J_{n}\right\}_{n \geq 1}$ is nonlattice, satisfying $\mathbb{E}\left[J^{\alpha^{*}}\right]=1, \alpha^{*}>0$ and $\left.\left(\mathbb{E}\left[J^{\alpha}\right]\right)^{\prime}\right|_{\alpha=\alpha^{*}}<\infty$, then

$$
\lim _{x \rightarrow \infty} \mathbb{P}\left[M_{\tau}>x\right] x^{\alpha^{*}}=\frac{\left(1-\left\|G_{+}\right\|\right)\left(1-\mathbb{E}\left[e^{-\alpha^{*} S_{\tau}}\right]\right)}{\alpha^{*} \int_{0}^{\infty} x e^{\alpha^{*} x} G_{+}(d x)} .
$$

Proof. The result is a direct consequence of Corollary 5.9 on p. 368 of [5].

\section{Main Results}

This section presents our main results in Theorems 3 and 4 . To avoid technical difficulties, we assume $\underline{\mu} \triangleq \inf _{j} \mu(j)>0$. With a small abuse of notation, as compared to the preceding Subsection [2.1, we redefine here $\Pi_{n}=\prod_{i=-n}^{-1} \mu\left(J_{i}\right), n \geq 1, \Pi_{0}=l$ and $M=\sup _{n \geq 0} \Pi_{n}$. In this paper we use the following standard notation. For any two real functions $a(t)$ and $b(t)$, we use $a(t)=o(b(t))$ to denote that $\lim _{t \rightarrow \infty} a(t) / b(t)=0$, and $a(t)=O(b(t))$ to denote that $\varlimsup_{t \rightarrow \infty} a(t) / b(t)<\infty$; when needed for increased clarity, we may explicitly write $a(t)=o(b(t))$ as $t \rightarrow \infty$.

Theorem 3. Assume that the process $\left\{\Pi_{n}\right\}$ satisfies the polynomial Gärtner-Ellis conditions (conditions 1) - 3) of Theorem 11), and $\sup _{j} \mathbb{E}\left[e^{\theta|B(j)-\mu(j)|}\right]<\infty$ for some $\theta>0$, then,

$$
\lim _{x \rightarrow \infty} \frac{\log \mathbb{P}[\Lambda>x]}{\log x}=\lim _{x \rightarrow \infty} \frac{\log \mathbb{P}[M>x]}{\log x}=-\alpha^{*} .
$$

Remark 5. Note that conditions 1) and 2) of Theorem 1 imply that there exists $j$ such that $\mu(j)>1$, since otherwise we have $\sup _{\alpha} \Psi(\alpha) \leq 0$, which would contradict $\Psi\left(\alpha^{*}\right)=0$ and $\Psi^{\prime}\left(\alpha^{*}\right)>0$ in condition 2). The following theorem covers the opposite situation when the previous condition is not satisfied, i.e., $\sup _{j} \mu(j)<1$.

Theorem 4. If $\sup _{j} \mu(j)<1$ and $\sup _{j} \mathbb{E}\left[e^{\theta|B(j)-\mu(j)|}\right]<\infty$ for some $\theta>0$, then,

$$
\lim _{x \rightarrow \infty} \frac{\log \mathbb{P}[\Lambda>x]}{\log x}=-\infty .
$$


Remark 6. Informally speaking, these two theorems show that the alternating periods of contractions and expansions, e.g., economic booms and recessions, are primarily responsible for the appearance of power law distributions; in other words, if there are no periods of expansions, i.e., the condition $\sup _{j} \mu(j)<1$ of Theorem 4 is satisfied, then $\Lambda$ has a tail that is lighter than any power law distribution. Furthermore, the first equality in (14) of Theorem 3 reveals a general asymptotic equivalence between the reflected modulated branching process and the corresponding reflected multiplicative process, showing that the power law exponent $\alpha^{*}$ is insensitive to the higher order distributional properties of $B(j)$ beyond the conditional mean $\mu(j)$.

Remark 7. A careful examination of the proofs reveals that the existence of a uniform upper bound of the exponential moments for $|B(j)-\mu(j)|$ could possibly be relaxed to $\sup _{j} \mathbb{E}\left[|B(j)-\mu(j)|^{\alpha}\right]<\infty$ for $\alpha>\alpha^{*}$. However, such an extension would considerably complicate the proofs. Furthermore, in most practical applications the distributions of $\{B(j)\}$ are typically very concentrated. For the preceding reasons, we do not consider such extensions.

We present the proofs of Theorems 3 and 4 in Subsection 6.1

\section{Exact Asymptotics}

This section presents the exact asymptotic approximations of the RMPs and RMBPs in the following two subsections, respectively.

\subsection{Exact Asymptotics of RMPs and the double Pareto phenomenon}

The following two theorems essentially provide a new general explanation of the measured double Pareto phenomenon (e.g., see [58, 67]) since they rely on two universal statistical laws: the first one being based on the large deviation theory and the latter being implied by the central limit theorem.

The theorems are direct translations from the corresponding queueing theory results. Theorem 5 is based on the large deviation result that studies the situation when $M$ is large, and Theorem [6 is derived from the heavy traffic approximation of a GI/GI/1 queue where we study the limiting behavior of a sequence of multiplicative processes with the multiplicative drift tending to one. These two theorems are basically corollaries of Theorem 5.2 in Chapter XIII and Theorem 7.1 in Chapter X of [5], respectively.

For a sequence of positive i.i.d. random variables $\left\{J, J_{n}\right\}_{n \geq 1}$, define $G_{+}$to be the ladder height distribution of the random walk $\left\{S_{n}=\sum_{i=1}^{n} \log J_{i}\right\}_{n \geq 1}$ with $\left\|G_{+}\right\|=\mathbb{P}\left[S_{n} \leq\right.$ 0 for all $n \geq 1$ ].

Theorem 5. If the sequence $\left\{\log J_{n}\right\}_{n \geq 1}$ is nonlattice, satisfying $\mathbb{E}\left[J^{\alpha^{*}}\right]=1, \alpha^{*}>0$, and $\left.\left(\mathbb{E}\left[J^{\alpha}\right]\right)^{\prime}\right|_{\alpha=\alpha^{*}}<\infty$, then

$$
\lim _{x \rightarrow \infty} \mathbb{P}[M>x] x^{\alpha^{*}}=\frac{1-\left\|G_{+}\right\|}{\alpha^{*} \int_{0}^{\infty} x e^{\alpha^{*} x} G_{+}(d x)} .
$$

Proof. The result is a direct consequence of Theorem 5.3 in Chapter XIII of [5].

Remark 8. If $S_{n}$ is lattice valued, see Remark 5.4 of Chapter XIII on p. 366 of [5].

Now, we study the limiting behavior of a sequence of multiplicative processes indexed by an integer $k$ where $J^{(k)}, S_{n}^{(k)}$ and $M^{(k)}$ are properly defined for all $k \geq 1$. 
Theorem 6. If $\left\{J^{(k)}, J_{n}^{(k)}\right\}_{n \geq 1}$ are positive and i.i.d. for each fixed $k$ with $m_{k} \triangleq \mathbb{E}\left[\log J^{(k)}\right]$, $\sigma_{k}^{2} \triangleq \operatorname{Var}\left[\log J^{(k)}\right]$, the random walks $\left\{S_{n}^{(k)}=\sum_{i=1}^{n} \log J_{i}^{(k)}\right\}_{n \geq 1}$ satisfy $m_{k}<0, \lim _{k \rightarrow \infty} m_{k}=$ $0, \underline{\lim }_{k \rightarrow \infty} \sigma_{k}^{2}>0$, and $\left(\log J^{(k)}\right)^{2}$ is uniformly integrable for all $k$, then, for $y \geq 1$,

$$
\lim _{k \rightarrow \infty} \mathbb{P}\left[\left(M^{(k)}\right)^{-m_{k} / \sigma_{k}^{2}}>y\right]=1 / y^{2} .
$$

Proof. From Theorem 7.1 in Chapter X on p. 287 of [5], we have, for $z \geq 0$,

$$
\lim _{k \rightarrow \infty} \mathbb{P}\left[-\frac{m_{k}}{\sigma_{k}^{2}} \log M^{(k)}>z\right]=e^{-2 z},
$$

which, by letting $z=\log y$, finishes the proof of Theorem 6 .

\subsection{Exact Asymptotics of Reflected Branching Processes}

In this subsection, assuming that $\left\{J, J_{n}\right\}_{n \geq 1}$ are i.i.d. and $\{\log \mu(J)\}$ is nonlattice, we will give an exact asymptotics for RMBPs using the implicit renewal theorem of Goldie (1991); see Theorem 2.3 and Corollary 2.4 in 32 . To this end, let $\left\{B(j), B^{i}(j)\right\}_{i, j}$ be independent random variables that are independent of $\left\{J, J_{n}\right\}$ and satisfy $B^{i}(j) \stackrel{d}{=} B(j)$.

Theorem 7. If $\sup _{j} \mathbb{E}\left[e^{\theta|B(j)-\mu(j)|}\right]<\infty$ for some $\theta>0, \mathbb{E}\left[\mu(J)^{\alpha^{*}}\right]=1, \alpha^{*}>0$ and $\mathbb{E}\left[\mu(J)^{\alpha^{*}+\delta}\right]<\infty$ for some $\delta>0$, then,

$$
\lim _{x \rightarrow \infty} \mathbb{P}[\Lambda>x] x^{\alpha^{*}}=\frac{\mathbb{E}\left[\left(\Lambda^{*}\right)^{\alpha^{*}}-(\mu(J) \Lambda)^{\alpha^{*}}\right]}{\alpha^{*} \mathbb{E}\left[\mu(J)^{\alpha^{*}} \log \mu(J)\right]},
$$

where $\Lambda^{*} \triangleq \max \left(\sum_{i=1}^{\Lambda} B^{i}(J), l\right)$ and $\Lambda$ is independent of $J$ and $\left\{B^{i}(j)\right\}_{i, j}$.

Remark 9. The preceding result is implicit because the constant on the right hand side of equation (16) involves the value of $\Lambda$, which is what we are trying to compute. In principle, to derive the explicit exact asymptotics for RMBPs is a difficult problem since the asymptotic constant depends on the behavior around the boundary $l$. However, in the scaling region where the boundary $l$ grows as well, albeit slowly, one can derive an explicit asymptotic characterization.

In the following, similarly as in Theorem 5 , we let $G_{+}$be the ladder height distribution of the nonlattice random walk $\left\{S_{n}=\sum_{i=1}^{n} \log \mu\left(J_{i}\right)\right\}_{n \geq 1}$ with $\left\|G_{+}\right\|=\mathbb{P}\left[S_{n} \leq 0\right.$ for all $\left.n \geq 1\right]<1$.

Theorem 8. If $\sup _{k} \mathbb{E}\left[e^{\theta|B(k)-\mu(k)|}\right]<\infty$ for some $\theta>0, \underline{\mu} \triangleq \inf _{j} \mu(j)>0, \mathbb{E}\left[\mu(J)^{\alpha^{*}}\right]=1$ for some $\alpha^{*}>0$ with $\left.\left(\mathbb{E}\left[J^{\alpha}\right]\right)^{\prime}\right|_{\alpha=\alpha^{*}}<\infty$, then, for any $\gamma>\overline{0}$,

$$
\lim _{\substack{l_{x}(\log x)^{3+\gamma} \\ x \rightarrow \infty}} \mathbb{P}\left[\Lambda^{l_{x}} / l_{x}>x\right] x^{\alpha^{*}}=\frac{1-\left\|G_{+}\right\|}{\alpha^{*} \int_{0}^{\infty} x e^{\alpha^{*} x} G_{+}(d x)} .
$$

The proofs of Theorems 7 and 8 are presented in Subsection 6.2. Here, we illustrate the exact asymptotics of the reflected branching process with the following simulation example.

Example 5. Assume that $\left\{J_{n}\right\}_{n \geq 1}$ is a Bernoulli process with $\mathbb{P}\left[J_{n}=1\right]=0.4=1-\mathbb{P}\left[J_{n}=0\right]$ and the i.i.d. random variables $\left\{B_{n}^{i}(1)\right\}_{i \geq 1},\left\{B_{n}^{i}(0)\right\}_{i \geq 1}$ follow Poisson distributions with means 1.5 and 0.6 , respectively. The simulation results of $10^{7}$ samples, for $l=1,5,13,21$, are drawn in Figure 4.2. From the figure we can clearly see that $\mathbb{P}\left[\Lambda^{l} / l \geq x\right]$ approaches the limiting value very quickly, i.e., for $l=13$ and $l=21$, the plots of $\mathbb{P}\left[\Lambda^{l} / l \geq x\right]$ are basically indistinguishable. 


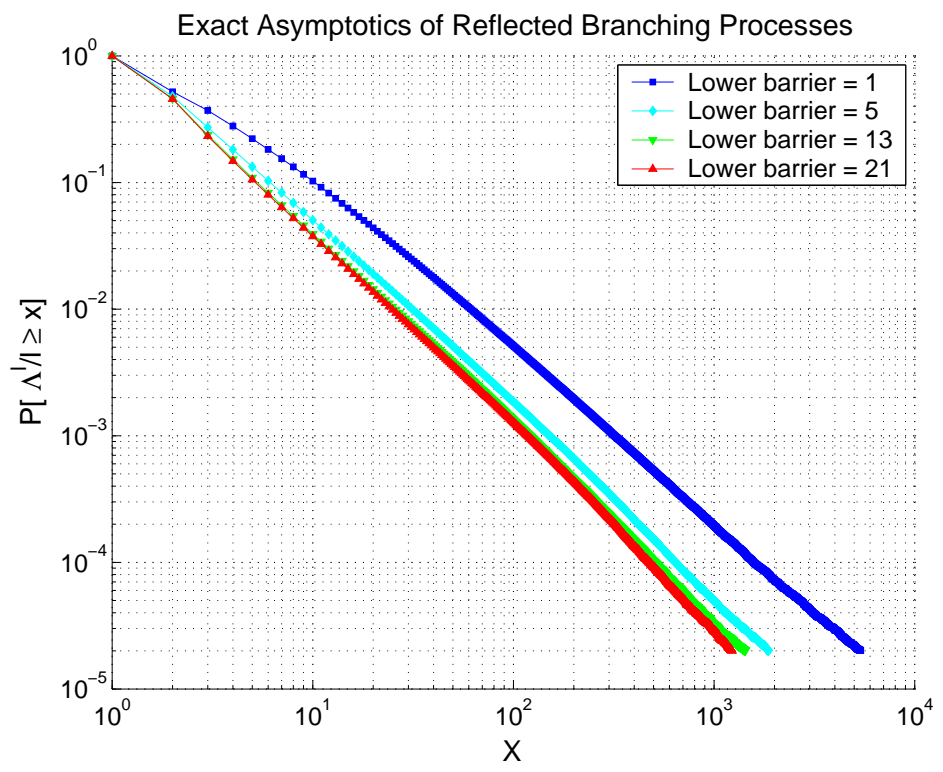

Figure 2: Simulation of $\mathbb{P}\left[\Lambda^{l} / l \geq x\right]$ versus $x$ parameterized by $l$.

\section{Discussion of Related Models}

Based on the study of reflected modulated branching processes, we address three related models: randomly stopped processes, modulated branching processes with absorbing barriers and truncated power laws.

\subsection{Randomly Stopped Processes}

In this subsection we discuss randomly stopped multiplicative and branching processes, respectively.

\subsubsection{Randomly Stopped Multiplicative Processes}

The following two theorems show that randomly stopped multiplicative processes and reflected multiplicative processes are intimately related and, to a certain extent, basically equivalent under more restrictive conditions. By following the approach of Chapter VIII of [5], we study the ladder heights of a multiplicative process. For any RMP with i.i.d positive multiplicative increments, the random variable $M$, as defined in Lemma 3. can be represented in terms of the ladder heights. To this end, define $\Pi_{n}^{0} \triangleq \prod_{i=0}^{n} J_{i}$ and the ladder height process $\left\{H_{i}\right\}_{i \geq 1}$ of $\left\{S_{n}=\sum_{i=1}^{n} \log J_{i}\right\}_{n \geq 1}$ with $\left\|G_{+}\right\|=\mathbb{P}\left[S_{n} \leq 0\right.$ for all $\left.n \geq 1\right]<1$ and $H_{i}^{e} \triangleq e^{H_{i}}$.

Theorem 9. Suppose that $\left\{J, J_{n}\right\}_{n \geq 1}$ is a positive i.i.d. sequence with $\mathbb{E}[\log J]<0$, then,

$$
M \stackrel{d}{=} \prod_{i=1}^{N} H_{i}^{e},
$$

where $N$ is independent of $\left\{H_{i}^{e}\right\}_{i \geq 1}$ and follows a geometric distribution $\mathbb{P}[N>n]=\left\|G_{+}\right\|^{n}$. 
Proof. Based on the well-known Pollaczek-Khinchin representation (see Chapter VIII of [5])

$$
\log M \stackrel{d}{=} \sum_{i=1}^{N} H_{i}
$$

where $N$ is independent of $\left\{H_{i}\right\}$ with $\mathbb{P}[N>n]=\left\|G_{+}\right\|^{n}$, it immediately follows that

$$
\mathbb{P}[M>x]=\mathbb{P}\left[e^{\sum_{i=1}^{N} H_{i}}>x\right]=\mathbb{P}\left[\prod_{i=1}^{N} H_{i}^{e}>x\right] .
$$

Conversely, we can prove that if the observation time has exponential tail, the stopped process has a power law tail under quite general conditions. Note that here we do not require $\left\{J_{n}\right\}$ to be an i.i.d. sequence.

Theorem 10. Let $N$ be an integer random variable independent of $\left\{J_{n}\right\}$ with

$$
\lim _{x \rightarrow \infty} \frac{\log \mathbb{P}[N>x]}{x}=-\lambda<0
$$

For a positive ergodic and stationary process $\left\{J_{n}\right\}_{n \geq 0}$, if $n^{-1} \log \mathbb{E}\left[\left(\Pi_{n}^{0}\right)^{\alpha}\right] \rightarrow \Psi(\alpha)<\infty$ as $n \rightarrow \infty$ in a neighborhood of $\alpha^{*}>0, \Psi(\alpha)$ is differentiable at $\alpha^{*}$ with $\Psi\left(\alpha^{*}\right)=\lambda, \Psi^{\prime}\left(\alpha^{*}\right)>0$ and $\mathbb{E}\left[\left(\Pi_{n}^{0}\right)^{\alpha^{*}}\right]<\infty$ for $n \geq 1$, then,

$$
\lim _{x \rightarrow \infty} \frac{\log \mathbb{P}\left[\Pi_{N}^{0}>x\right]}{\log x}=-\alpha^{*}
$$

The proof of Theorem 10 is presented in Subsection 6.3 .

Remark 10. This theorem generalizes the previous results from [34, 64, 66] where only i.i.d. multiplicative increments are considered.

Actually the following theorem shows that randomly stopped multiplicative processes and reflected multiplicative processes are basically equivalent under more restrictive conditions. This equivalence is established using classical results on $M / G I / 1$ queue. In this regard, we assume that $\left\{J_{n}\right\}_{n \geq 1}$ is an i.i.d. process, $\Pi_{n}^{0}$ is the corresponding multiplicative process, $N$ is a geometric random variable that is independent of $\Pi_{n}^{0}$ with $\mathbb{P}[N>n]=\rho^{n}, 0<\rho<1$, and $\bar{G}(t), t \geq 0$ is a complementary cumulative distribution function.

Theorem 11. If a randomly stopped multiplicative process $\Pi_{N}^{0}$ satisfies

$$
\mathbb{P}\left[\log J_{1} \leq x\right]=\int_{0}^{x} \bar{G}(y) d y / \int_{0}^{\infty} \bar{G}(y) d y, x \geq 0
$$

for some $\bar{G}(\cdot)$, then, we can always construct a stationary $R M P$ such that $\Pi_{N}^{0} \stackrel{d}{=} M$. Furthermore, if in addition $\int_{0}^{\infty} e^{\alpha^{*} y} \bar{G}(y) d y=\rho^{-1} \int_{0}^{\infty} \bar{G}(y) d y, \int_{0}^{\infty} y e^{\alpha^{*} y} \bar{G}(y) d y<\infty$ for $\alpha^{*}>0$, then,

$$
\lim _{x \rightarrow \infty} \mathbb{P}[M>x] x^{\alpha^{*}}=\lim _{x \rightarrow \infty} \mathbb{P}\left[\Pi_{N}^{0}>x\right] x^{\alpha^{*}}=\frac{(1-\rho) \int_{0}^{\infty} \bar{G}(y) d y}{\alpha^{*} \rho \int_{0}^{\infty} y e^{\alpha^{*} y} \bar{G}(y) d y} .
$$

The proof of Theorem 11 is presented in Subsection 6.3. 


\subsubsection{Randomly Stopped Branching Processes}

In the following theorem, we extend Theorem 10 of the preceding subsection to the context of randomly stopped branching processes. Define $\Pi_{n}^{0} \triangleq \prod_{i=0}^{n} \mu\left(J_{i}\right)$.

Theorem 12. Suppose that $N$ is independent of $B_{n}^{i}(j) \geq 1$ for all $n, i, j$. Then, under the same conditions as in Theorem 10 with $\mathbb{E}\left[\left(\Pi_{n}^{0}\right)^{\alpha}\right]<\infty$ for $n \geq 1$ and $\Psi(\alpha)$ being differentiable in a neighborhood of $\alpha^{*}>0$, we obtain, for $\left\{Z_{n}\right\}_{n \geq 0}$ defined in (1) with a bounded initial value $Z_{0}<z_{0}<\infty$,

$$
\lim _{x \rightarrow \infty} \frac{\log \mathbb{P}\left[Z_{N}>x\right]}{\log x}=\lim _{x \rightarrow \infty} \frac{\log \mathbb{P}\left[\Pi_{N}^{0}>x\right]}{\log x}=-\alpha^{*} .
$$

The proof of this theorem is based on similar arguments as in the proof of Theorem 3, and we defer it to Subsection 6.3.

\subsection{Branching Processes with Absorbing Barriers}

For many dynamic processes, e.g., city sizes, quite often when the sizes of the objects fall below a threshold, the whole object disappears, e.g., urban decay. Therefore, it is natural to study branching processes with absorbing barriers. As already discussed in Subsection 2.2. we know that a single object with an absorbing barrier can result in power law distributions based on the duality with the queueing cycle maximum. In this context, we can also study a more complicated situation where the newly generated objects can join the system and evolve together. This naturally models the arrivals to popular Web sites (hotspots), since information (news) is distributed according to a branching process, e.g., user $A$ passes the information to $B$ and $C$; further $B$ may inform $D$, etc. Empirical examination shows that Web requests follow power law distributions, e.g., see [35, 1].

For a lower barrier $l>0$ and the modulated branching process $\left\{Z_{n}^{l}\right\}_{n \geq 1}$ with $Z_{0}^{l}=l$ specified in Definition 1, define stopping time $P \triangleq \inf \left\{n>0: Z_{n}^{l} \leq l\right\}$, where the modulating process $\left\{J, J_{n}\right\}$ is a sequence of i.i.d. random variables. This branching process, denoted by $Z_{P}$, vanishes completely after $P$; it is easy to prove that $\mathbb{E}[P]<\infty$ when $\mathbb{E}\left[\log \mu\left(J_{0}\right)\right]<0$.

Let the arrivals $\left\{A_{n}\right\}_{n>-\infty}$ be a sequence of i.i.d. Poisson random variables with $\mathbb{E}\left[A_{n}\right]=$ $q>0$ that is independent of other random variables. At time $n, A_{n}$ objects are generated and join the system, each evolving according to an i.i.d. copy of the modulated branching process $Z_{P}$. Suppose that the system has reached its stationarity with $N_{n}$ objects being in the system at time $n$, and then, by Little's Law, $\mathbb{E}\left[N_{n}\right]=q \mathbb{E}[P]$. Furthermore, assume that object $j$ observed at time $n=0$, if any, is generated at time $\left(-P_{j}^{r}\right)$ with a size $Z_{-P_{j}^{r}}^{l}$, where the random variables $\left\{P_{j}^{r}\right\}$ are i.i.d. and follow the equilibrium distribution of $P$. Then, the total size of all objects $Z_{s}$ observed at time $n=0$ in stationarity can be represented as

$$
Z_{s}=\sum_{j=1}^{N_{0}} Z_{-P_{j}^{r}}^{l} .
$$

Next, we show that $Z_{s}$ follows a power law. The proof of the following theorem is essentially a corollary of Theorem 3 .

Theorem 13. Under the conditions described in this subsection, if $\left\{\mu\left(J_{n}\right)\right\}$ satisfies $\inf _{j} \mu(j)>$ $0, \mathbb{E}[\log \mu(J)]<0, \mathbb{E}\left[\mu(J)^{\alpha^{*}}\right]=1$ for some $\alpha^{*}>0, \mathbb{E}\left[\mu(J)^{\alpha^{*}+\delta}\right]<\infty$ for some $\delta>0$, and $\sup _{j} \mathbb{E}\left[e^{\theta|B(j)-\mu(j)|}\right]<\infty$ for some $\theta>0$, then,

$$
\lim _{x \rightarrow \infty} \frac{\log \mathbb{P}\left[Z_{s}>x\right]}{\log x}=-\alpha^{*}
$$

The proof of this theorem is presented in Subsection 6.4 . 


\subsection{Truncated Power Laws}

Truncated power laws have been observed empirically in many practical situations where the studied objects have natural upper boundaries. Here, we want to point out that by using the duality between the modulated branching processes and the queueing theory, one easily obtains truncated power laws when adding both a lower and an upper barrier to the modulated branching process. To illustrate this point, recall that $M / M / 1 / b$ queue with a finite buffer $b$ results in a truncated geometric distribution for the number of customers in the queue, and by the duality, it essentially follows that in a proportional growth world with both a lower and an upper barrier, truncated power laws can naturally arise, playing a similar role as truncated exponential/geometric distributions do in an additive world. Prior related work on this subject can be found in [71].

\section{Proofs}

\subsection{Proofs of Theorems 3 and 4}

The proof of Theorem 3, composed of the upper bound and the lower bound, and the proof of Theorem 4 are presented in the following three subsections, respectively.

\subsubsection{Proof of Theorem 3; Upper Bound}

Since the proof is based on the change (increase) of boundary $l$, we denote this dependence explicitly as $\Lambda^{l} \equiv \Lambda$. According to Lemma 2, the initial value of $\left\{\Lambda_{n}\right\}$ has no impact on $\Lambda$ and, therefore, in this subsection we simply assume that $\Lambda_{0}^{l}=l$. Before stating the proof of the upper bound, we establish preliminary Lemmas 4, 5, 6, and 7.

The first lemma shows that, most likely, the supremum of $Z_{n}$ occurs for an index $n \leq x$.

Lemma 4. For any $\beta>0$, the branching process $Z_{n}^{l}$ defined in (1) satisfies

$$
\sum_{n>x}^{\infty} \mathbb{P}\left[Z_{n}^{l}>x\right]=O\left(\frac{1}{x^{\beta}}\right) \text { as } x \rightarrow \infty .
$$

Proof. Similarly as in the proof of Lemma 1, note that for $\Pi_{n-1}^{0}=\prod_{i=0}^{n-1} \mu\left(J_{i}\right)$, the stochastic process $W_{n}=Z_{n}^{l} / \Pi_{n-1}^{0}, n \geq 1$ is a martingale with respect to the filtration $\mathcal{F}_{n}=\sigma\left(J_{i}, Z_{i}, 0 \leq\right.$ $i \leq n-1)$ that satisfies $\mathbb{E}\left[W_{1}\right]=1$. Therefore, by recalling $\Pi_{n}=\prod_{i=-n}^{-1} \mu\left(J_{i}\right)$, we obtain, for any $\varepsilon>0$,

$$
\begin{aligned}
\mathbb{P}\left[Z_{n}^{l}>x\right] & =\mathbb{P}\left[W_{n} \Pi_{n-1}^{0}>x\right]=\mathbb{P}\left[\left(W_{n} e^{-\varepsilon n}\right)\left(\Pi_{n-1}^{0} e^{\varepsilon n}\right)>x\right] \\
& \leq \mathbb{P}\left[W_{n} e^{-\varepsilon n}>1\right]+\mathbb{P}\left[\Pi_{n} e^{\varepsilon n}>x\right] \\
& \leq \mathbb{E}\left[W_{n} e^{-\varepsilon n}\right]+\mathbb{P}\left[\Pi_{n} e^{\varepsilon n}>x\right] .
\end{aligned}
$$

Next, by using the martingale property $\mathbb{E}\left[W_{n}\right]=\mathbb{E}\left[W_{1}\right]=1$, we derive

$$
\sum_{n>x}^{\infty} \mathbb{E}\left[W_{n} e^{-\varepsilon n}\right]=\sum_{n>x}^{\infty} e^{-\varepsilon n} \leq \frac{e^{-\varepsilon x}}{1-e^{-\varepsilon}}=O\left(\frac{1}{x^{\beta}}\right) \quad \text { as } x \rightarrow \infty .
$$

Then, recalling conditions 1) and 2) of Theorem 3 (or Theorem 11), we can choose $\delta, \varepsilon>$ 0 small enough and $n_{0}$ large enough such that $\Psi\left(\alpha^{*}-\delta\right)+2 \varepsilon\left(\alpha^{*}-\delta\right)=-\zeta<0$ and 
$n^{-1} \log \mathbb{E}\left[\Pi_{n}^{\left(\alpha^{*}-\delta\right)}\right]<\Psi\left(\alpha^{*}-\delta\right)+\varepsilon\left(\alpha^{*}-\delta\right)$ for $n>n_{0}$, which implies, for $x>n_{0}$,

$$
\begin{aligned}
\sum_{n>x}^{\infty} \mathbb{P}\left[\Pi_{n} e^{\varepsilon n}>x\right] & \leq \sum_{n>x}^{\infty} \frac{\mathbb{E}\left[\Pi_{n}^{\left(\alpha^{*}-\delta\right)}\right] e^{\varepsilon\left(\alpha^{*}-\delta\right) n}}{x^{\left(\alpha^{*}-\delta\right)}} \leq \sum_{n>x}^{\infty} \frac{e^{-\zeta n}}{x^{\alpha^{*}-\delta}} \\
& \leq \frac{e^{-\zeta x}}{\left(1-e^{-\zeta}\right) x^{\alpha^{*}-\delta}}=O\left(\frac{1}{x^{\beta}}\right) \text { as } x \rightarrow \infty .
\end{aligned}
$$

Finally, by using (19), (20) and (21), we complete the proof.

The following lemma relates $\Lambda_{n}$ to the corresponding multiplicative process.

Lemma 5. Let $\varepsilon>0$ and $\Lambda_{n}^{l}$ be the reflected branching process, as defined in (2), then, for $x \geq l$,

$$
\mathbb{P}\left[\Lambda_{n}^{l}>x\right] \leq \mathbb{P}\left[\max _{1 \leq j \leq n} \Pi_{j}(1+\varepsilon)^{j}>x / l\right]+n \mathbb{P}\left[\mathcal{B}_{0}^{l, \varepsilon}\right]
$$

where $\Pi_{j}=\prod_{i=-j}^{-1} \mu\left(J_{i}\right)$ and $\mathcal{B}_{n}^{l, \varepsilon}=\bigcup_{j \geq l}\left\{\sum_{i=1}^{j} B_{n}^{i}\left(J_{n}\right)>j \mu\left(J_{n}\right)(1+\varepsilon)\right\}$ for any integer $n$.

Proof. From (6), we have

$$
\Lambda_{n}^{l} \stackrel{d}{=} \max \left(Z_{-n}^{l}, Z_{-(n-1)}^{l}, \cdots, Z_{-1}^{l}, Z_{0}^{l}\right) .
$$

Next, let $Z_{-n}^{l}(k)$ be the branching process that starts at time $-n$ with $l$ objects and is observed at time $k \geq-n$. Note that $Z_{-i}^{l}(-i)=l, Z_{-i}^{l}(0) \equiv Z_{-i}^{l}$ and

$$
Z_{-j}^{l}=\sum_{i=1}^{Z_{-j}^{l}(-1)} B_{-1}^{i}\left(J_{-1}\right)
$$

for $j \geq 1$. Now, by using the preceding observation, (22) and $Z_{0}^{l}=l$, we derive, for $x \geq l$,

$$
\begin{aligned}
\mathbb{P} & {\left[\Lambda_{n}^{l}>x\right] \leq \mathbb{P}\left[\max \left(Z_{-n}^{l}, Z_{-(n-1)}^{l}, \cdots, Z_{-1}^{l}, l\right)>x,\left(\mathcal{B}_{-1}^{l, \varepsilon}\right)^{C}\right]+\mathbb{P}\left[\mathcal{B}_{-1}^{l, \varepsilon}\right] } \\
\leq & \mathbb{P}\left[\max \left(Z_{-n}^{l}(-1)(1+\varepsilon) \mu\left(J_{-1}\right), \cdots, Z_{-2}^{l}(-1)(1+\varepsilon) \mu\left(J_{-1}\right), l(1+\varepsilon) \mu\left(J_{-1}\right)\right)>x,\left(\mathcal{B}_{-1}^{l, \varepsilon}\right)^{C}\right] \\
& +\mathbb{P}\left[\mathcal{B}_{-1}^{l, \varepsilon}\right] \\
& \leq \mathbb{P}\left[\left\{(1+\varepsilon) \mu\left(J_{-1}\right) \max _{2 \leq i \leq n}\left(Z_{i}^{l}(-1)\right)>x\right\} \bigcup\left\{\mu\left(J_{-1}\right)(1+\varepsilon)>x / l\right\}\right]+\mathbb{P}\left[\mathcal{B}_{-1}^{l, \varepsilon}\right] .
\end{aligned}
$$

Then, intersecting with event $\mathcal{B}_{-2}^{l, \varepsilon}$ and using

$$
Z_{-j}^{l}(-1)=\sum_{i=1}^{Z_{-j}^{l}(-2)} B_{-2}^{i}\left(J_{-2}\right)
$$

for $j \geq 2$, one easily obtains

$$
\begin{aligned}
\mathbb{P}\left[\Lambda_{n}^{l}>x\right] \leq \mathbb{P}[ & \left\{(1+\varepsilon)^{2} \mu\left(J_{-2}\right) \mu\left(J_{-1}\right) \max _{3 \leq i \leq n}\left(Z_{i}^{l}(-2)\right)>x\right\} \\
& \left.\bigcup\left\{\max \left(\mu\left(J_{-2}\right) \mu\left(J_{-1}\right)(1+\varepsilon)^{2}, \mu\left(J_{-1}\right)(1+\varepsilon)\right)>x / l\right\}\right] \\
+ & \mathbb{P}\left[\mathcal{B}_{-2}^{l, \varepsilon}\right]+\mathbb{P}\left[\mathcal{B}_{-1}^{l, \varepsilon}\right],
\end{aligned}
$$

which, by continuing the induction and using $\mathbb{P}\left[\mathcal{B}_{i}^{l, \varepsilon}\right]=\mathbb{P}\left[\mathcal{B}_{0}^{l, \varepsilon}\right]$ for all $i$, finishes the proof. 
Now, we show that the "error" event $\mathcal{B}_{0}^{l, \varepsilon}$ in the preceding lemma has a negligible probability for large $l$ relative to any power law distribution.

Lemma 6. If $\sup _{j} \mathbb{E}\left[e^{\theta|B(j)-\mu(j)|}\right]<\infty, \theta>0$ and $\underline{\mu}=\inf _{j} \mu(j)>0$, then, by setting $l_{x}=\left\lfloor x^{\delta}\right\rfloor, 0<\delta<1$ in the definition of $\mathcal{B}_{0}^{l_{x}, \varepsilon}$ in Lemma $\overline{5}$, we obtain, for any $\beta>0$,

$$
\mathbb{P}\left[\mathcal{B}_{0}^{l_{x}, \varepsilon}\right]=O\left(\frac{1}{x^{\beta}}\right) \quad \text { as } \quad x \rightarrow \infty
$$

Proof. First, we derive

$$
\begin{aligned}
P(n) & \triangleq \mathbb{P}\left[\sum_{i=1}^{n} B_{0}^{i}\left(J_{0}\right)>\mu\left(J_{0}\right)(1+\varepsilon) n\right] \\
& \leq \mathbb{P}\left[\sum_{i=1}^{n}\left(B_{0}^{i}\left(J_{0}\right)-\mu\left(J_{0}\right)\right)>\varepsilon \underline{\mu} n\right] \\
& \leq\left(\mathbb{E}\left[e^{\zeta(B(J)-\mu(J))}\right]\right)^{n} e^{-\zeta \varepsilon \underline{\mu} n}, \quad \zeta>0,
\end{aligned}
$$

which, by using the elementary inequality $e^{x} \leq 1+x+x^{2} e^{|x|} / 2, x \in \mathbb{R}$ and setting $x=$ $B(J)-\mu(J)$, yields

$$
P(n) \leq\left(1+\frac{\zeta^{2}}{2} \mathbb{E}\left[(B(J)-\mu(J))^{2} e^{\zeta|B(J)-\mu(J)|]}\right)^{n} e^{-\zeta \varepsilon \underline{\mu} n} .\right.
$$

For any $\eta>0$ and large enough $n$ such that $\zeta=\eta \log n /(\varepsilon \underline{\mu} n)<\theta$, the assumption $\sup _{j} \mathbb{E}\left[e^{\theta|B(j)-\mu(j)|}\right]<\infty$ implies

$$
C \triangleq \mathbb{E}\left[(B(J)-\mu(J))^{2} e^{\zeta|B(J)-\mu(J)|}\right]<\infty
$$

which yields

$$
P(n) \leq\left(1+\frac{C(\eta \log n)^{2}}{2(n \varepsilon \underline{\mu})^{2}}\right)^{n} n^{-\eta}=O\left(\frac{1}{n^{\eta}}\right)
$$

Therefore, choosing $\eta=1+\beta / \delta$ in (23), we obtain, for $l_{x}=\left\lfloor x^{\delta}\right\rfloor, 0<\delta<1$ and $\beta>0$, as $x \rightarrow \infty$

$$
\mathbb{P}\left[\mathcal{B}_{0}^{l_{x}, \varepsilon}\right] \leq \sum_{i=l_{x}}^{\infty} P(n) \leq O\left(\sum_{n=\left\lfloor x^{\delta}\right\rfloor}^{\infty} \frac{1}{n^{\eta}}\right)=O\left(\frac{1}{x^{\beta}}\right)
$$

The following lemma allows us to increase the lower barrier in order to prove the upper bound.

Lemma 7. Assume that $\Lambda_{n}^{l_{1}}$ and $\Lambda_{n}^{l_{2}}$ are defined on the same sequence $\left\{B_{n}^{j}\left(J_{n}\right)\right\}$ with initial conditions $l_{1}$ and $l_{2}$, respectively. If $l_{1} \geq l_{2}$, then, for all $n \geq 0$,

$$
\Lambda_{n}^{l_{1}} \geq \Lambda_{n}^{l_{2}}
$$


Proof. The result holds trivially for $n=0$. Now we prove the result using induction. Suppose that it is true for all $0 \leq k \leq n$, and for $k=n+1$,

$$
\Lambda_{n+1}^{l_{1}}=\max \left(\sum_{i=1}^{\Lambda_{n}^{l_{1}}} B_{n}^{i}\left(J_{n}\right), l_{1}\right) \geq \max \left(\sum_{i=1}^{\Lambda_{n}^{l_{2}}} B_{n}^{i}\left(J_{n}\right), l_{2}\right)=\Lambda_{n+1}^{l_{2}},
$$

which implies that the lemma is true for all $n \geq 0$.

Now, we are ready to complete the proof of the upper bound.

Proof of the upper bound of Theorem [3: Choosing $l_{x}=\left\lfloor x^{\varepsilon}\right\rfloor \geq l, 0<\varepsilon<1$, using Lemma 7 and then Lemma 5, we derive

$$
\begin{aligned}
\mathbb{P}\left[\Lambda^{l}>x\right] & =\mathbb{P}\left[\sup _{j \geq 1} Z_{-j}^{l}>x\right] \leq \mathbb{P}\left[\Lambda_{\lfloor x\rfloor}^{l}>x\right]+\mathbb{P}\left[\sup _{j>x} Z_{-j}^{l}>x\right] \\
& \leq \mathbb{P}\left[\Lambda_{\lfloor x\rfloor}^{l_{x}}>x\right]+\sum_{j>x} \mathbb{P}\left[Z_{j}^{l}>x\right] \\
& \leq \mathbb{P}\left[\sup _{j \geq 1} \Pi_{j}(1+\varepsilon)^{j}>x^{1-\varepsilon}\right]+x \mathbb{P}\left[\mathcal{B}_{0}^{l_{x}, \varepsilon}\right]+\sum_{j>x} \mathbb{P}\left[Z_{j}^{l}>x\right] \\
& \triangleq I_{1}(x)+I_{2}(x)+I_{3}(x) .
\end{aligned}
$$

Now, define a new process $\left\{\mu^{\varepsilon}\left(J_{n}\right)=\mu\left(J_{n}\right)(1+\varepsilon)\right\}_{n \geq 1}$ and $\Pi_{n}^{\varepsilon}=\prod_{i=-n}^{-1} \mu^{\varepsilon}\left(J_{i}\right)$. Then, for $\varepsilon$ small enough, we have

1) $n^{-1} \log \mathbb{E}\left(\Pi_{n}^{\varepsilon}\right)^{\alpha} \rightarrow \Psi^{\varepsilon}(\alpha)=\Psi(\alpha)+\alpha \log (1+\varepsilon)$ as $n \rightarrow \infty$ for $\left|\alpha-\alpha^{*}\right|<\varepsilon^{*}$,

2) $\Psi^{\varepsilon}$ is finite in a neighborhood of $\alpha_{\varepsilon}^{*}, \alpha_{\varepsilon}^{*}<\alpha^{*}$, and differentiable at $\alpha_{\varepsilon}^{*}$ with $\Psi\left(\alpha_{\varepsilon}^{*}\right)+$ $\alpha_{\varepsilon}^{*} \log (1+\varepsilon)=0, \Psi^{\prime}\left(\alpha_{\varepsilon}^{*}\right)>0$, and

3) $\mathbb{E}\left[\left(\Pi_{n}^{\varepsilon}\right)^{\alpha_{\varepsilon}^{*}}\right]<\infty$ for $n \geq 1$.

Therefore, by Theorem 1, we obtain

$$
\lim _{x \rightarrow \infty} \frac{\log \mathbb{P}\left[\sup _{i \geq 1} \Pi_{i}(1+\varepsilon)^{i}>x^{1-\varepsilon}\right]}{\log x}=-(1-\varepsilon) \alpha_{\varepsilon}^{*},
$$

which, in conjunction with Lemma 4 and Lemma 6, yields

$$
I_{2}(x)+I_{3}(x)=o\left(I_{1}(x)\right) .
$$

Then, combining (24), (25) and (26) yields

$$
\frac{\log \mathbb{P}\left[\Lambda^{l}>x\right]}{\log x} \leq \frac{\log \left((1+o(1)) I_{1}(x)\right)}{\log x} \longrightarrow-(1-\varepsilon) \alpha_{\varepsilon}^{*} \quad \text { as } x \rightarrow \infty .
$$

Since $\Psi^{\varepsilon}(\alpha)$ is continuous in a neighborhood of $\alpha^{*}$ in both $\alpha$ and $\varepsilon$, we derive

$$
\lim _{\varepsilon \rightarrow 0} \alpha_{\varepsilon}^{*}=\alpha^{*}
$$

implying,

$$
\varlimsup_{x \rightarrow \infty} \frac{\log \mathbb{P}[\Lambda>x]}{\log x} \leq-\alpha^{*}
$$




\subsubsection{Proof of Theorem 3; Lower Bound}

In order to prove the lower bound, we need to establish the following three lemmas. Specifically, Corollary 1 allows us to obtain a lower bound for $\Lambda$ while, maybe somewhat counterintuitively, increasing the lower barrier $l$.

Lemma 8. Let $\left\{\Lambda_{n}^{y_{1}}\right\}$ and $\left\{\Lambda_{n}^{y_{2}}\right\}$ be defined on the same modulating sequence $\left\{J_{n}\right\}_{n \geq 0}$ and independent random variables $\left\{B_{n}^{i, 1}(j), B_{n}^{i, 2}(j)\right\}$ with $B_{n}^{i, k}(j)$ identically distributed for fixed $j$. Then,

$$
\Lambda_{n}^{y_{1}+y_{2}} \stackrel{d}{\leq} \Lambda_{n}^{y_{1}}+\Lambda_{n}^{y_{2}}
$$

where $\Lambda_{n}^{y_{1}}$ and $\Lambda_{n}^{y_{2}}$ are conditionally independent given $\left\{J_{n}\right\}_{n \geq 0}$.

Proof. We use induction to prove this lemma. Starting with $n=1$, we obtain

$$
\begin{aligned}
\Lambda_{1}^{y_{1}+y_{2}} & =\max \left(\sum_{i=1}^{y_{1}+y_{2}} B_{0}^{i}\left(J_{0}\right), y_{1}+y_{2}\right) \stackrel{d}{=} \max \left(\sum_{i=1}^{y_{1}} B_{0}^{i, 1}\left(J_{0}\right)+\sum_{i=1}^{y_{2}} B_{0}^{i, 2}\left(J_{0}\right), y_{1}+y_{2}\right) \\
& \leq \max \left(\sum_{i=1}^{y_{1}} B_{0}^{i, 1}\left(J_{0}\right), y_{1}\right)+\max \left(\sum_{i=1}^{y_{2}} B_{0}^{i, 2}\left(J_{0}\right), y_{2}\right) \\
& \stackrel{d}{=} \Lambda_{1}^{y_{1}}+\Lambda_{1}^{y_{2}}
\end{aligned}
$$

since for any $x_{1}, x_{2}, y_{1}, y_{2}$,

$$
\max \left(x_{1}+x_{2}, y_{1}+y_{2}\right) \leq \max \left(x_{1}, y_{1}\right)+\max \left(x_{2}, y_{2}\right) .
$$

The proof is completed by induction in $n$,

$$
\begin{aligned}
\Lambda_{n+1}^{y_{1}+y_{2}} & =\max \left(\sum_{i=1}^{\Lambda_{n}^{y_{1}+y_{2}}} B_{1}^{i}\left(J_{n}\right), y_{1}+y_{2}\right) \stackrel{d}{\leq} \max \left(\sum_{i=1}^{\Lambda_{n}^{y_{1}}+\Lambda_{n}^{y_{2}}} B_{1}^{i}\left(J_{n}\right), y_{1}+y_{2}\right) \\
& \stackrel{d}{\leq} \Lambda_{n+1}^{y_{1}}+\Lambda_{n+1}^{y_{2}} .
\end{aligned}
$$

Next, a straightforward application of the preceding lemma yields the following corollary.

Corollary 1. If $\left\{\Lambda_{n, j}^{1}\right\}_{1 \leq j \leq y}$ are conditionally i.i.d copies of $\Lambda_{n}^{1}$ given $\left\{J_{i}\right\}_{1 \leq i \leq n}$, then,

$$
\Lambda_{n}^{y}{ }^{d} \sum_{j=1}^{y} \Lambda_{n, j}^{1}
$$

Now, we basically establish that the supremum of $\Pi_{i}$ occurs most likely for small indexes $i \leq h \log x$.

Lemma 9. Assume that condition 1) of Theorem 3 is satisfied, then, for $0 \leq \varepsilon<1$ and any $\beta>0$, there exists $h>0$ such that, when $x \rightarrow \infty$,

$$
\mathbb{P}\left[\sup _{i>h \log x} \Pi_{i}(1-\varepsilon)^{i}>x\right]=O\left(\frac{1}{x^{\beta}}\right) .
$$


Proof. Using condition 1) of Theorem 3, we can choose $0<\alpha<\alpha^{*}$ with $n^{-1} \log \mathbb{E}\left[\Pi_{n}^{\alpha}\right] \rightarrow$ $\Psi(\alpha)<0$ and $n_{0}$ large enough, such that $\mathbb{E}\left[\Pi_{n}^{\alpha}\right]<\zeta^{n}, 0<\zeta<1, n>n_{0}$. Thus, for $h=-\beta / \log \zeta>0$ and $x>e^{n_{0} / h}$,

$$
\mathbb{P}\left[\sup _{i>h \log x} \Pi_{i}(1-\varepsilon)^{i}>x\right] \leq \sum_{i>h \log x}^{\infty} \mathbb{P}\left[\Pi_{i}>x\right] \leq \sum_{i>h \log x}^{\infty} \frac{\mathbb{E}\left[\Pi_{i}^{\alpha}\right]}{x^{\alpha}} \leq \sum_{i>h \log x}^{\infty} \frac{\zeta^{i}}{x^{\alpha}}=O\left(\frac{1}{x^{\beta}}\right) .
$$

Finally, the last lemma shows that $\sum_{i=1}^{j} B_{n}^{i}\left(J_{n}\right)$ can not deviate too much from $j \mu\left(J_{n}\right)$ for large $j$.

Lemma 10. For $0<\delta, \varepsilon<1$ and $\mathcal{C}_{n}^{l, \varepsilon} \triangleq \bigcup_{j \geq l}\left\{\sum_{i=1}^{j} B_{n}^{i}\left(J_{n}\right)<j \mu\left(J_{n}\right)(1-\varepsilon)\right\}$, we obtain, for any $\beta>0$,

$$
\mathbb{P}\left[\mathcal{C}_{0}^{\left\lfloor x^{\delta}\right\rfloor, \varepsilon}\right]=O\left(\frac{1}{x^{\beta}}\right)
$$

Proof. The proof of this lemma is basically the same as Lemma 6, Observe

$$
\begin{aligned}
P(n) & \triangleq \mathbb{P}\left[\sum_{i=1}^{n} B_{0}^{i}\left(J_{0}\right)<\mu\left(J_{0}\right)(1-\varepsilon) n\right] \\
& \leq \mathbb{P}\left[\sum_{i=1}^{n}\left(B_{0}^{i}\left(J_{0}\right)-\mu\left(J_{0}\right)\right)<-\varepsilon \mu\left(J_{0}\right) n\right] \\
& \leq \mathbb{P}\left[\sum_{i=1}^{n}\left(\mu\left(J_{0}\right)-B_{0}^{i}\left(J_{0}\right)\right)>\varepsilon \underline{\mu} n\right] .
\end{aligned}
$$

Then, by using a similar argument as in deriving (23), we can prove, for any $\beta>0$,

$$
\mathbb{P}\left[\mathcal{C}_{0}^{\left\lfloor x^{\delta}\right\rfloor, \varepsilon}\right]=O\left(\frac{1}{x^{\beta}}\right)
$$

Next, we can complete the proof of the lower bound of Theorem 3 .

Proof of the lower bound of Theorem [3: First, using Corollary 1, we obtain, for any integer $y \geq 1$,

$$
\mathbb{P}\left[\Lambda_{n}^{l}>x\right] \geq \mathbb{P}\left[\Lambda_{n}^{1}>x\right]=\frac{y \mathbb{P}\left[\Lambda_{n}^{1}>x\right]}{y} \geq \frac{\mathbb{P}\left[\sum_{j=1}^{y} \Lambda_{n, j}^{1}>y x\right]}{y} \geq \frac{\mathbb{P}\left[\Lambda_{n}^{y}>y x\right]}{y} .
$$

Now, using (16), similarly as in the proof of Lemma 15, for $0<\varepsilon<1$ and $\mathcal{C}_{n}^{l, \varepsilon}$ defined in 
Lemma 10, we derive

$$
\begin{aligned}
\mathbb{P}\left[\Lambda_{n}^{y}>y x\right] & \geq \mathbb{P}\left[\max _{0 \leq i \leq n}\left(Z_{-i}^{y x}\right)>y x,\left(\bigcup_{i=-n}^{-1} \mathcal{C}_{i}^{y, \varepsilon}\right)^{C}\right] \\
& \geq \mathbb{P}\left[\sup _{1 \leq i \leq n} \Pi_{i}(1-\varepsilon)^{i}>x,\left(\bigcup_{i=-n}^{-1} \mathcal{C}_{i}^{y, \varepsilon}\right)^{C}\right] \\
& \geq \mathbb{P}\left[\sup _{1 \leq i \leq n} \Pi_{i}(1-\varepsilon)^{i}>x\right]-n \mathbb{P}\left[\mathcal{C}_{0}^{y, \varepsilon}\right] \\
& \geq \mathbb{P}\left[\sup _{i \geq 1} \Pi_{i}(1-\varepsilon)^{i}>x\right]-\mathbb{P}\left[\sup _{i>n} \Pi_{i}(1-\varepsilon)^{i}>x\right]-n \mathbb{P}\left[\mathcal{C}_{0}^{y, \varepsilon}\right] \\
& \triangleq I_{1}(x)-I_{2}(x)-I_{3}(x) ;
\end{aligned}
$$

note that $\left\{I_{j}(x)\right\}_{1 \leq j \leq 3}$ here are different from those in (24).

Next, similarly as in the proof of the upper bound, define a new process $\left\{\mu_{\varepsilon}\left(J_{n}\right)=\mu\left(J_{n}\right)(1-\right.$ $\varepsilon)\}_{n \geq 1}$ and let $\Pi_{n}^{\varepsilon}=\prod_{i=-n}^{-1} \mu_{\varepsilon}\left(J_{i}\right)$. Then, for $\varepsilon$ small enough, we have

1) $n^{-1} \log \mathbb{E}\left(\Pi_{n}^{\varepsilon}\right)^{\alpha} \rightarrow \Psi^{\varepsilon}(\alpha)=\Psi(\alpha)+\alpha \log (1-\varepsilon)$ as $n \rightarrow \infty$ for $\left|\alpha-\alpha^{*}\right|<\varepsilon^{*}$,

2) $\Psi^{\varepsilon}(\alpha)$ is finite in a neighborhood of $\alpha_{\varepsilon}^{*}, \alpha_{\varepsilon}^{*}>\alpha^{*}$ and differentiable at $\alpha_{\varepsilon}^{*}$ with $\Psi\left(\alpha_{\varepsilon}^{*}\right)+$ $\alpha_{\varepsilon}^{*} \log (1-\varepsilon)=0, \Psi^{\prime}\left(\alpha_{\varepsilon}^{*}\right)>0$, and

3) $\mathbb{E}\left[\left(\Pi_{n}^{\varepsilon}\right)^{\alpha_{\varepsilon}^{*}}\right]<\infty$ for $n \geq 1$.

Therefore, by Theorem 1, we obtain

$$
\lim _{x \rightarrow \infty} \frac{\log \mathbb{P}\left[\sup _{i \geq 1} \Pi_{i}(1-\varepsilon)^{i}>x\right]}{\log x}=-\alpha_{\varepsilon}^{*} .
$$

Now, by setting $y=\left\lfloor x^{\delta}\right\rfloor, 0<\delta<1, n=\lfloor x\rfloor$ in (28), (29), and using Lemmas 9 and 10, it is easy to see that

$$
I_{2}(x)+I_{3}(x)=o\left(I_{1}(x)\right)
$$

which, by (28) and (29), yields

$$
\begin{aligned}
\log \mathbb{P}[\Lambda>x] & \geq \log \mathbb{P}\left[\Lambda_{n}^{l}>x\right] \\
& \geq \log \left(I_{1}(x)-I_{2}(x)-I_{3}(x)\right)-\delta \log x \\
& =\log \left((1-o(1)) I_{1}(x)\right)-\delta \log x
\end{aligned}
$$

From the preceding inequality and (30), we obtain

$$
\varliminf_{x \rightarrow \infty} \frac{\log \mathbb{P}[\Lambda>x]}{\log x} \geq-\alpha_{\varepsilon}^{*}-\delta
$$

Since $\Psi^{\varepsilon}(\alpha)$ is continuous in a neighborhood of $\alpha^{*}$ in both $\alpha$ and $\varepsilon$, we have $\lim _{\varepsilon \rightarrow 0} \alpha_{\varepsilon}^{*}=\alpha^{*}$. Then, passing $\varepsilon, \delta \rightarrow 0$ in (31) completes the proof of the lower bound, which, in conjunction with (27), finishes the proof of Theorem 3 . 


\subsubsection{Proof of Theorem 4}

Proof. Using the same arguments as in deriving (24) in the proof of the upper bound of Theorem 3, we obtain, for $l_{x}=\lfloor x\rfloor \geq l$ and $0<\varepsilon<1$,

$$
\begin{aligned}
\mathbb{P}[\Lambda>x] & \leq \mathbb{P}\left[\Lambda_{\lfloor x\rfloor}^{l}>x\right]+\mathbb{P}\left[\sup _{j>x} Z_{-j}^{l}>x\right] \\
& \leq \mathbb{P}\left[\Lambda_{\lfloor x\rfloor}^{l_{x}}>x\right]+\sum_{j>x} \mathbb{P}\left[Z_{j}^{l}>x\right] \\
& \leq \mathbb{P}\left[\sup _{j \geq 1} \Pi_{j}(1+\varepsilon)^{j}>1\right]+x \mathbb{P}\left[\mathcal{B}_{0}^{l_{x}, \varepsilon}\right]+\sum_{j>x}^{\infty} \mathbb{P}\left[Z_{j}^{l}>x\right] \\
& \triangleq I_{1}(x)+I_{2}(x)+I_{3}(x) .
\end{aligned}
$$

Recalling $\Pi_{j}=\prod_{i=-1}^{-j} \mu\left(J_{i}\right)$ and noting $\sup _{j} \mu(j)<1$, we can choose $\varepsilon>0$ such that $\sup _{j} \mu(j)(1+\varepsilon)<1$, which implies $I_{1}(x)=0$. And, using Lemma $\underline{6}$, we obtain $I_{2}(x)=O\left(x^{-\beta}\right)$ for all $\beta>0$.

Next, using similar arguments as in deriving (19) in the proof of Lemma 4, we obtain, for $\varepsilon>0$ and $j \geq 1$,

$$
\mathbb{P}\left[Z_{j}^{l}>x\right] \leq \mathbb{E}\left[W_{j} e^{-\varepsilon j}\right]+\mathbb{P}\left[\Pi_{j} e^{\varepsilon j}>x\right],
$$

which, by recalling $\sup _{j} \mu(j)<1$ and choosing $\varepsilon$ small enough such that $\mathbb{P}\left[\Pi_{j} e^{\varepsilon j}>x\right]=0$ for $x>1$, yields,

$$
I_{3}(x) \leq \sum_{j>\lfloor x\rfloor}^{\infty} \mathbb{E}\left[W_{j} e^{-\varepsilon j}\right]=\sum_{j>\lfloor x\rfloor}^{\infty} e^{-\varepsilon j}=O\left(e^{-\varepsilon x}\right) .
$$

Finally, combining (32) and the bounds on $I_{1}(x), I_{2}(x)$ and $I_{3}(x)$ finishes the proof.

\subsection{Proofs of Theorems 7 and 8}

\subsubsection{Proof of Theorem 7}

In order to prove Theorem 7, we first derive the following lemma.

Lemma 11. Under the assumptions of Theorem 7 , there exists $\gamma>0$, such that

$$
\mathbb{E}\left[\left|\sum_{i=1}^{\Lambda}\left(B^{i}(J)-\mu(J)\right)\right|^{\alpha^{*}+\gamma}\right]<\infty .
$$

Proof. We observe that, for $x>0$,

$$
\begin{aligned}
\mathbb{P}\left[\left|\sum_{i=1}^{\Lambda}\left(B^{i}(J)-\mu(J)\right)\right|>x\right] & =\mathbb{P}\left[\sum_{i=1}^{\Lambda}\left(B^{i}(J)-\mu(J)\right)>x\right]+\mathbb{P}\left[\mu(J)-\sum_{i=1}^{\Lambda}\left(B^{i}(J)\right)>x\right] \\
& \triangleq I_{1}+I_{2} .
\end{aligned}
$$

We choose $\gamma$ and $\delta$ such that $0<\gamma<\alpha^{*}$ and $\gamma<\delta<\alpha^{*}$. To evaluate $I_{1}$, for $0<\varepsilon<$ $\left(\alpha^{*}-\delta\right) / 2$, we set $0<\beta \triangleq(\delta+\varepsilon) /\left(\alpha^{*}-\varepsilon\right)<1$, and obtain

$$
I_{1} \leq \mathbb{P}\left[\Lambda>x^{1+\beta}\right]+\mathbb{P}\left[\sum_{i=1}^{\Lambda}\left(B^{i}(J)-\mu(J)\right)>x, \Lambda \leq x^{1+\beta}\right],
$$


which, by recalling that Theorem 3 implies $\mathbb{P}[\Lambda>x]=O\left(x^{-\left(\alpha^{*}-\varepsilon\right)}\right)$, results in

$$
\mathbb{P}\left[\Lambda>x^{1+\beta}\right]=O\left(x^{-\left(\alpha^{*}+\delta\right)}\right) .
$$

Now, we study the second probability on the right-hand side of (35). Using the fact that $J$ is independent of $\Lambda$ and applying Chernoff bound, we obtain for $\zeta>0$,

$$
\begin{aligned}
\mathbb{P}\left[\sum_{i=1}^{\Lambda}\left(B^{i}(J)-\mu(J)\right)>x, \Lambda \leq x^{1+\beta}\right] & =\sum_{n=l}^{\left\lfloor x^{1+\beta}\right\rfloor} \mathbb{P}[\Lambda=n] \mathbb{P}\left[\sum_{i=1}^{n}\left(B^{i}(J)-\mu(J)\right)>x\right] \\
& \leq \sum_{n=l}^{\left\lfloor x^{1+\beta}\right\rfloor} \mathbb{P}[\Lambda=n] e^{-\zeta x}\left(\mathbb{E}\left[e^{\zeta(B(J)-\mu(J))}\right]\right)^{n} .
\end{aligned}
$$

Then, setting $t=\zeta(B(J)-\mu(J))$ in (35), using $e^{t} \leq 1+t+t^{2} e^{|t|} / 2, t \in \mathbb{R}$ and observing that $\mathbb{E}[B(J)-\mu(J)]=0$, (35) is further upper bounded by

$$
\sum_{n=l}^{\left\lfloor x^{1+\beta}\right\rfloor} \mathbb{P}[\Lambda=n]\left(1+\frac{\zeta^{2}}{2} \mathbb{E}\left[(B(J)-\mu(J))^{2} e^{\zeta|B(J)-\mu(J)|]}\right]\right)^{n} e^{-\zeta x} .
$$

Next, $\sup _{j} \mathbb{E}\left[e^{\theta|B(j)-\mu(j)|}\right]<\infty$ implies $C \triangleq \mathbb{E}\left[(B(J)-\mu(J))^{2} e^{\zeta|B(J)-\mu(J)|}\right]<\infty, \zeta<\theta$. Hence, for $x$ large, we have $\zeta=\left(\alpha^{*}+\delta\right) \log x / x<\theta$, implying that (35) is bounded by

$$
\sum_{n=1}^{\left\lfloor x^{1+\beta}\right\rfloor} \mathbb{P}[\Lambda=n]\left(1+\frac{C\left(\left(\alpha^{*}+\delta\right) \log x\right)^{2}}{2 x^{2}}\right)^{x^{1+\beta}} x^{-\left(\alpha^{*}+\delta\right)}=O\left(x^{-\left(\alpha^{*}+\delta\right)}\right)
$$

since $\beta<1$. Combining (34), (35) and (36) proves

$$
I_{1}=O\left(x^{-\left(\alpha^{*}+\delta\right)}\right) \text {. }
$$

By using the same approach as in proving (37), we can also show

$$
I_{2}=O\left(x^{-\left(\alpha^{*}+\delta\right)}\right) \text {, }
$$

implying

$$
\mathbb{P}\left[\left|\sum_{i=1}^{\Lambda}\left(B^{i}(J)-\mu(J)\right)\right|>x\right]=O\left(x^{-\left(\alpha^{*}+\delta\right)}\right) .
$$

Therefore, since $\delta>\gamma$,

$$
\begin{aligned}
\mathbb{E}\left[\left|\sum_{i=1}^{\Lambda}\left(B^{i}(J)-\mu(J)\right)\right|^{\alpha^{*}+\gamma}\right] & =\int_{0}^{\infty} \mathbb{P}\left[\left|\sum_{i=1}^{\Lambda}\left(B^{i}(J)-\mu(J)\right)\right|^{\alpha^{*}+\gamma}>x\right] d x \\
& =O\left(\int_{0}^{\infty} x^{-\frac{\alpha^{*}+\delta}{\alpha^{*}+\gamma}} d x\right)<\infty,
\end{aligned}
$$

which finishes the proof of the lemma.

Now, we proceed with proving Theorem 7 . 
Proof of Theorem [7. The proof is based on Corollary 2.4 in [32], for which it is sufficient to show

$$
I \triangleq \mathbb{E}\left[\left|\left(\max \left(\sum_{i=1}^{\Lambda} B^{i}(J), l\right)\right)^{\alpha^{*}}-(\mu(J) \Lambda)^{\alpha^{*}}\right|\right]<\infty .
$$

In order to prove the preceding inequality, we will use the following elementary inequality (see equation (9.27) in [32]), for $x, y \geq 0$,

$$
\left|x^{\alpha}-y^{\alpha}\right| \leq \begin{cases}|x-y|^{\alpha}, & 0<\alpha \leq 1 \\ \alpha|x-y|\left(x^{\alpha-1}+y^{\alpha-1}\right), & 1<\alpha<\infty .\end{cases}
$$

First, we prove the case when $0<\alpha^{*} \leq 1$. Using the fact that

$$
\begin{array}{rl}
\left(\max \left(\sum_{i=1}^{\Lambda} B^{i}(J), l\right)\right)^{\alpha^{*}}=l^{\alpha^{*}} & \mathbf{1}\left(\sum_{i=1}^{\Lambda} B^{i}(J) \leq l\right)+\left(\sum_{i=1}^{\Lambda} B^{i}(J)\right)^{\alpha^{*}} \\
& -\left(\sum_{i=1}^{\Lambda} B^{i}(J)\right)^{\alpha^{*}} \mathbf{1}\left(\sum_{i=1}^{\Lambda} B^{i}(J) \leq l\right),
\end{array}
$$

we obtain, by using (39) and recalling Lemma 11,

$$
\begin{aligned}
I & \leq 2 l^{\alpha^{*}}+\mathbb{E}\left[\left|\left(\sum_{i=1}^{\Lambda} B^{i}(J)\right)^{\alpha^{*}}-(\mu(J) \Lambda)^{\alpha^{*}}\right|\right] \\
& \leq 2 l^{\alpha^{*}}+\mathbb{E}\left[\left|\sum_{i=1}^{\Lambda}\left(B^{i}(J)-\mu(J)\right)\right|^{\alpha^{*}}\right]<\infty .
\end{aligned}
$$

Next, we prove the case when $\alpha^{*}>1$. Applying (39) and (40) in (38), we obtain

$$
\begin{aligned}
I & \leq 2 l^{\alpha^{*}}+\mathbb{E}\left[\left|\left(\sum_{i=1}^{\Lambda} B^{i}(J)\right)^{\alpha^{*}}-(\mu(J) \Lambda)^{\alpha^{*}}\right|\right] \\
& \leq 2 l^{\alpha^{*}}+\alpha^{*} \mathbb{E}\left[\left|\sum_{i=1}^{\Lambda}\left(B^{i}(J)-\mu(J)\right)\right|\left|\sum_{i=1}^{\Lambda} B^{i}(J)\right|^{\alpha^{*}-1}\right] \\
& +\alpha^{*} \mathbb{E}\left[\left|\sum_{i=1}^{\Lambda}\left(B^{i}(J)-\mu(J)\right)\right||\Lambda \mu(J)|^{\alpha^{*}-1}\right] \\
& \triangleq 2 l^{\alpha^{*}}+I_{1}+I_{2} .
\end{aligned}
$$

For $I_{1}$, we use Hölder's inequality to obtain, for $0<\varepsilon<1 / 2$,

$$
\begin{aligned}
I_{1} \leq \alpha^{*} \mathbb{E}\left[\left|\sum_{i=1}^{\Lambda}\left(B^{i}(J)-\mu(J)\right)\right|^{\frac{\alpha^{*}}{1-\varepsilon}}\right]^{\frac{1-\varepsilon}{\alpha^{*}}} \mathbb{E}\left[\left(\sum_{i=1}^{\Lambda} B^{i}(J)\right)^{\frac{\left(\alpha^{*}-1\right) \alpha^{*}}{\alpha^{*}+\varepsilon-1}}\right]^{\frac{\alpha^{*}+\varepsilon-1}{\alpha^{*}}} \\
\leq \alpha^{*} \mathbb{E}\left[\left|\sum_{i=1}^{\Lambda}\left(B^{i}(J)-\mu(J)\right)\right|^{\frac{\alpha^{*}}{1-\varepsilon}}\right]^{\frac{1-\varepsilon}{\alpha^{*}}} \mathbb{E}\left[\Lambda^{\frac{\left(\alpha^{*}-1\right) \alpha^{*}}{\alpha^{*}+\varepsilon-1}}\right]^{\frac{\alpha^{*}+\varepsilon-1}{\alpha^{*}}}
\end{aligned}
$$


where the last inequality uses the fact that

$$
\sum_{i=1}^{\Lambda} B^{i}(J) \stackrel{d}{\leq} \Lambda
$$

Now, Theorem 3 implies that

$$
\mathbb{E}\left[\Lambda^{\frac{\left(\alpha^{*}-1\right) \alpha^{*}}{\alpha^{*}+\varepsilon-1}}\right]<\infty
$$

which, in combination with Lemma 11 and (43), results in

$$
I_{1}<\infty
$$

Using the same argument as in proving (43), we obtain, by noting that $\Lambda$ and $\mu(J)$ are independent from each other,

$$
I_{2} \leq \alpha^{*} \mathbb{E}\left[\left|\sum_{i=1}^{\Lambda}\left(B^{i}(J)-\mu(J)\right)\right|^{\frac{\alpha^{*}}{1-\varepsilon}}\right]^{\frac{1-\varepsilon}{\alpha^{*}}} \mathbb{E}\left[\Lambda^{\frac{\left(\alpha^{*}-1\right) \alpha^{*}}{\alpha^{*}+\varepsilon-1}}\right]^{\frac{\alpha^{*}+\varepsilon-1}{\alpha^{*}}} \mathbb{E}\left[\mu(J)^{\frac{\left(\alpha^{*}-1\right) \alpha^{*}}{\alpha^{*}+\varepsilon-1}}\right]^{\frac{\alpha^{*}+\varepsilon-1}{\alpha^{*}}}<\infty
$$

which, in conjunction with (44), proves (38) and finishes the proof of Theorem 7.

\subsubsection{Proof of Theorem 8}

The proof of this theorem relies on the following lemmas; the first one is based on Theorem 3.7.1 of [24].

Lemma 12. $\left\{X(j), X_{i}(j)\right\}_{i, j \in \mathbb{Z}}$ are zero mean independent random variables that are identically distributed for fixed $j$. Fix a sequence $a_{n} \rightarrow 0$ such that $n a_{n} \rightarrow \infty$ as $n \rightarrow \infty$. If $\sup _{j} \mathbb{E}\left[e^{\theta X(j)}\right]<\infty$ for $\theta>0$, then, there exist $n_{0}, h>0$, such that for all $n>n_{0}$ and any random variable $J \in \mathbb{Z}$,

$$
\mathbb{P}\left[\sum_{i=1}^{n} X_{i}(J)>\sqrt{\frac{n}{a_{n}}}\right] \leq e^{-\frac{h}{a_{n}}}
$$

Proof. For $0<\beta<\theta$, define $\varphi_{J}(\omega)=\mathbb{E}\left[e^{\omega X(J)} \mid J\right]$ and use Taylor expansion to derive

$$
\varphi_{J}(\omega)=\varphi_{J}(0)+\varphi_{J}^{\prime}(0) \omega+\frac{\varphi_{J}^{\prime \prime}(\zeta)}{2 !} \omega^{2}, 0<\zeta<\omega \leq \beta
$$

Noting that $\varphi_{J}(0)=1, \varphi_{J}^{\prime}(0)=0$ and

$$
\varphi_{J}^{\prime \prime}(\zeta)=\mathbb{E}\left[X(J)^{2} e^{\zeta X(J)} \mid J\right] \leq \sup _{j} \mathbb{E}\left[X(j)^{2} e^{\beta X(j)}\right] \triangleq K_{\beta}<\infty
$$

we obtain, for $0<\omega \leq \beta$,

$$
\varphi_{J}(\omega) \leq 1+K_{\beta} \omega^{2},
$$

which implies,

$$
\mathbb{P}\left[\sum_{i=1}^{n} X_{i}(J)>\sqrt{\frac{n}{a_{n}}}\right] \leq e^{-\omega \sqrt{\frac{n}{a_{n}}}} \mathbb{E}\left[\left(\varphi_{J}(\omega)\right)^{n}\right] \leq e^{-\omega \sqrt{\frac{n}{a_{n}}}}\left(1+K_{\beta} \omega^{2}\right)^{n} \leq e^{-\omega \sqrt{\frac{n}{a_{n}}}} e^{n K_{\beta} \omega^{2}} .
$$


Since there exists $n_{0}$ such that $\beta>1 /\left(2 K_{\beta} \sqrt{n a_{n}}\right)$ for all $n>n_{0}$, we can choose $\omega=$ $1 /\left(2 K_{\beta} \sqrt{n a_{n}}\right)$, which implies, for $n>n_{0}$,

$$
\mathbb{P}\left[\sum_{i=1}^{n} X_{i}(J)>\sqrt{\frac{n}{a_{n}}}\right] \leq e^{-\frac{1}{4 K_{\beta} a_{n}}}=e^{-\frac{h}{a_{n}}},
$$

where $h=1 /\left(4 K_{\beta}\right)>0$.

Lemma 13. For any $l \in \mathbb{N}$, define

$$
\mathscr{D}_{n}^{l} \triangleq \bigcup_{k \geq l}\left\{\sum_{i=1}^{k} B_{n}^{i}\left(J_{n}\right)>k \mu\left(J_{n}\right)\left(1+\underline{\mu}^{-1} l^{-\frac{1}{3}}\right)\right\}
$$

and

$$
\mathscr{E}_{n}^{l} \triangleq \bigcup_{k \geq l}\left\{\sum_{i=1}^{k} B_{n}^{i}\left(J_{n}\right)<k \mu\left(J_{n}\right)\left(1-\underline{\mu}^{-1} l^{-\frac{1}{3}}\right)\right\} .
$$

If $l \geq(\log x)^{3+\gamma}, \gamma>0$, then, under the conditions of Theorem $\mathbb{8}$, we obtain, for any $\beta>0$,

$$
\mathbb{P}\left[\mathscr{D}_{n}^{l}\right]=O\left(\frac{1}{x^{\beta}}\right) \text { and } \mathbb{P}\left[\mathscr{E}_{n}^{l}\right]=O\left(\frac{1}{x^{\beta}}\right)
$$

Proof. Defining $a_{n}=n^{-1 / 3}$ and observing that $n a_{n}$ is monotonically increasing in $n$, we obtain

$$
\begin{aligned}
\mathbb{P}\left[\mathscr{D}_{n}^{l}\right] & \leq \sum_{k=l}^{\infty} \mathbb{P}\left[\sum_{i=1}^{k} B_{n}^{i}\left(J_{n}\right)>k \mu\left(J_{n}\right)\left(1+\frac{1}{\underline{\mu}} \sqrt{\frac{1}{l a_{l}}}\right)\right] \\
& \leq \sum_{k=l}^{\infty} \mathbb{P}\left[\sum_{i=1}^{k} B_{n}^{i}\left(J_{n}\right)>k \mu\left(J_{n}\right)\left(1+\frac{1}{\underline{\mu}} \sqrt{\frac{1}{k a_{k}}}\right)\right] \\
& \leq \sum_{k=l}^{\infty} \mathbb{P}\left[\sum_{i=1}^{k}\left(B_{n}^{i}\left(J_{n}\right)-\mu\left(J_{n}\right)\right)>\sqrt{\frac{k}{a_{k}}}\right]
\end{aligned}
$$

which, by applying Lemma 12, yields

$$
\mathbb{P}\left[\mathscr{D}_{n}^{l}\right] \leq \sum_{k=l}^{\infty} e^{-\frac{h}{a_{k}}} \leq \sum_{k \geq(\log x)^{3+\gamma}}^{\infty} e^{-h k^{1 / 3}}=O\left(\frac{1}{x^{\beta}}\right) \text { as } x \rightarrow \infty .
$$

By the same argument,

$$
\begin{aligned}
\mathbb{P}\left[\mathscr{E}_{n} l\right] & \leq \sum_{k=l}^{\infty} \mathbb{P}\left[\sum_{i=1}^{k} B_{n}^{i}\left(J_{n}\right)<k \mu\left(J_{n}\right)\left(1-\frac{1}{\underline{\mu}} \sqrt{\frac{1}{l \cdot a_{l}}}\right)\right] \\
& \leq \sum_{k=l}^{\infty} \mathbb{P}\left[\sum_{i=1}^{k}\left(\mu\left(J_{n}\right)-B_{n}^{i}\left(J_{n}\right)\right)>\sqrt{\frac{k}{a_{k}}}\right] \\
& =O\left(\frac{1}{x^{\beta}}\right) \text { as } x \rightarrow \infty .
\end{aligned}
$$


Following the proof of Lemma 4 with minor modifications, we can prove the following stronger result.

Lemma 14. For any $\beta>0$, there exists $h>0$ such that the branching process defined in (1) satisfies

$$
\sum_{n \geq h \log x}^{\infty} \mathbb{P}\left[Z_{n}^{l}>x\right]=O\left(\frac{1}{x^{\beta}}\right) \text { as } x \rightarrow \infty .
$$

Now, we proceed with the proof of Theorem 8 .

Proof (of Theorem 8): First, we establish the upper bound. Setting $\varepsilon=\underline{\mu}^{-1} l^{-1 / 3}$ in Lemma 5, we obtain

$$
\mathbb{P}\left[\Lambda_{n}^{l}>l x\right] \leq \mathbb{P}\left[\max _{1 \leq j \leq n} \Pi_{j}\left(1+\underline{\mu}^{-1} l^{-\frac{1}{3}}\right)^{j}>x\right]+n \mathbb{P}\left[\mathscr{D}_{0}^{l}\right],
$$

where $\mathscr{D}_{0}^{l}$ is defined in Lemma 13, For $l \geq(\log x)^{3+\gamma}$, we obtain

$$
\begin{aligned}
\mathbb{P}\left[\Lambda^{l}>l x\right] & =\mathbb{P}\left[\sup _{j \geq 1} Z_{-j}>l x\right] \leq \mathbb{P}\left[\Lambda_{n}^{l}>l x\right]+\mathbb{P}\left[\sup _{j>n} Z_{-j}>l x\right] \\
& \leq \mathbb{P}\left[\sup _{1 \leq j \leq n} \Pi_{j}\left(1+\underline{\mu}^{-1} l^{-\frac{1}{3}}\right)^{j}>x\right]+n \mathbb{P}\left[\mathscr{D}_{0}^{l}\right]+\sum_{j>n}^{\infty} \mathbb{P}\left[Z_{j}^{l}>l x\right],
\end{aligned}
$$

which, by setting $n=\lfloor h \log x\rfloor$ with $h$ being chosen as in Lemma 14 and applying Lemmas 13 , 14. yields,

$$
\begin{aligned}
\mathbb{P}\left[\Lambda^{l}>l x\right] & \leq \mathbb{P}\left[\sup _{1 \leq j \leq h \log x} \Pi_{j}\left(1+\underline{\mu}^{-1} l^{-\frac{1}{3}}\right)^{j}>x\right]+o\left(\frac{1}{x^{\alpha^{*}}}\right) \\
& \leq \mathbb{P}\left[\sup _{j \geq 1} \Pi_{j}>x\left(1+\frac{1}{\underline{\mu}(\log x)^{1+\gamma / 3}}\right)^{-h \log x}\right]+o\left(\frac{1}{x^{\alpha^{*}}}\right) .
\end{aligned}
$$

Finally, by using Theorem 5 and observing that $\lim _{x \rightarrow \infty}\left(1+\frac{1}{\underline{\mu}(\log x)^{1+\gamma / 3}}\right)^{-h \log x}=1$, we obtain

$$
\varlimsup_{\substack{l \geq(\log x)^{3+\gamma} \\ x \rightarrow \infty}} \mathbb{P}\left[\Lambda^{l} / l>x\right] x^{\alpha^{*}} \leq \frac{1-\left\|G_{+}\right\|}{\alpha^{*} \int_{0}^{\infty} x e^{\alpha^{*} x} G_{+}(d x)} .
$$

Next, we prove the lower bound. Recall that $\Pi_{n}^{i} \triangleq \prod_{j=i}^{n-1} \mu\left(J_{j}\right)$ and $\Pi_{i} \triangleq \prod_{j=-1}^{-i} \mu\left(J_{j}\right)$. Then, for $l \geq(\log x)^{3+\gamma}$ and $n=\lfloor h \log x\rfloor$ where $h$ is chosen as in Lemma 14, we have

$$
\begin{aligned}
\mathbb{P}\left[\Lambda_{n}^{l}>l x\right] & \geq \mathbb{P}\left[\sup _{0 \leq i \leq n-1} \Pi_{n}^{i}\left(1-\underline{\mu}^{-1} l^{-\frac{1}{3}}\right)^{n-i}>x\right]-\mathbb{P}\left[\mathscr{E}_{0} l\right]-\cdots-\mathbb{P}\left[\mathscr{E}_{n-1}^{l}\right] \\
& =\mathbb{P}\left[\sup _{1 \leq i \leq n} \Pi_{i}\left(1-\underline{\mu}^{-1} l^{-\frac{1}{3}}\right)^{i}>x\right]-n \mathbb{P}\left[\mathscr{E}_{0}^{l}\right] \\
& \geq \mathbb{P}\left[\sup _{i \geq 1} \Pi_{i}\left(1-\underline{\mu}^{-1} l^{-\frac{1}{3}}\right)^{h \log x}>x\right]-\mathbb{P}\left[\sup _{i>n} \Pi_{i}\left(1-\underline{\mu}^{-1} l^{-\frac{1}{3}}\right)^{i}>x\right]-n \mathbb{P}\left[\mathscr{E}_{0}^{l}\right] \\
& \triangleq I_{1}-I_{2}-I_{3},
\end{aligned}
$$


where $\mathscr{E}_{0}$ is defined in Lemma 13. By Lemma 9, we obtain,

$$
I_{2} \leq \mathbb{P}\left[\sup _{i>h \log x} \Pi_{i}>x\right]=o\left(\frac{1}{x^{\alpha^{*}}}\right)
$$

and by Lemma 13 ,

$$
I_{3}=o\left(\frac{1}{x^{\alpha^{*}}}\right)
$$

Thus, combining (45), (46) and (47), we obtain

$$
\mathbb{P}\left[\Lambda_{n}^{l}>l x\right] \geq \mathbb{P}\left[\sup _{j \geq 1} \Pi_{j}\left(1-\frac{1}{\underline{\mu}(\log x)^{1+\gamma / 3}}\right)^{h \log x}>x\right]-o\left(\frac{1}{x^{\alpha^{*}}}\right),
$$

which, by using the same argument as in the proof of the upper bound, yields

$$
\varliminf_{l \geq \underset{x \rightarrow \infty}{(\log x)^{3+\gamma}}} \mathbb{P}\left[\Lambda^{l} / l>x\right] x^{\alpha^{*}} \geq \frac{1-\left\|G_{+}\right\|}{\alpha^{*} \int_{0}^{\infty} x e^{\alpha^{*} x} G_{+}(d x)} .
$$

\subsection{Proofs of Theorems 10,11 and 12}

Proof of Theorem [10. First, we prove the upper bound. For a fixed $\alpha$ that is in the neighborhood of $\alpha^{*}$ and $0<\varepsilon<\lambda$, there exists $n_{\varepsilon}$ such that $\mathbb{E}\left[\left(\Pi_{n}^{0}\right)^{\alpha}\right]<e^{(\Psi(\alpha)+\varepsilon) n}$ and $e^{-(\lambda-\varepsilon) n}>$ $\mathbb{P}[N \geq n]>e^{-(\lambda+\varepsilon) n}$ for all $n \geq n_{\varepsilon}$. Since $\Psi\left(\alpha^{*}\right)=\lambda$ and $\Psi^{\prime}\left(\alpha^{*}\right)>0$, we can choose $\delta, \varepsilon>0$ small enough such that $\Psi\left(\alpha^{*}-\delta\right)-\lambda+2 \varepsilon=-\xi<0$. Thus, noting that $N$ is independent of $\Pi_{n}$, we obtain

$$
\begin{aligned}
\mathbb{P}\left[\Pi_{N}^{0}>x\right] & =\sum_{n=1}^{\infty} \mathbb{P}[N=n] \mathbb{P}\left[\Pi_{n}^{0}>x\right] \\
& \leq \sum_{n=1}^{n_{\varepsilon}} \mathbb{P}[N=n] \mathbb{P}\left[\Pi_{n}^{0}>x\right]+\sum_{n=n_{\varepsilon}}^{\infty} \mathbb{P}[N \geq n] \mathbb{P}\left[\Pi_{n}^{0}>x\right] \\
& \leq \sum_{n=1}^{n_{\varepsilon}} \mathbb{P}[N=n] \frac{\mathbb{E}\left[\left(\Pi_{n}^{0}\right)^{\alpha^{*}}\right]}{x^{\alpha^{*}}}+\sum_{n=n_{\varepsilon}}^{\infty} e^{-(\lambda-\varepsilon) n} \frac{\mathbb{E}\left[\left(\Pi_{n}^{0}\right)^{\alpha^{*}-\delta}\right]}{x^{\alpha^{*}-\delta}} \\
& \leq O\left(\frac{1}{x^{\alpha^{*}}}\right)+\frac{1}{x^{\alpha^{*}-\delta}} \sum_{n=n_{\varepsilon}}^{\infty} e^{-\xi n},
\end{aligned}
$$

which implies

$$
\varlimsup_{x \rightarrow \infty} \frac{\log \mathbb{P}\left[\Pi_{N}^{0}>x\right]}{\log x}=-\alpha^{*}+\delta
$$

Passing $\delta \rightarrow 0$ in the preceding equality completes the proof of the upper bound.

Next, we prove the lower bound by using the standard exponential change of measure argument. For $0<3 \varepsilon<\lambda, \delta>2 \varepsilon /(\lambda-3 \varepsilon)$ and $\log x>n_{\varepsilon}$, recalling that $e^{-(\lambda-\varepsilon) n}>\mathbb{P}[N \geq$ $n]>e^{-(\lambda+\varepsilon) n}$, we obtain, for large $x$,

$$
\begin{aligned}
\mathbb{P}\left[\frac{(1+\delta) \log x}{\Psi^{\prime}\left(\alpha^{*}\right)} \leq N \leq \frac{(1+2 \delta) \log x}{\Psi^{\prime}\left(\alpha^{*}\right)}\right] & \geq e^{-\frac{(\lambda+\varepsilon)(1+\delta) \log x}{\Psi^{\prime}\left(\alpha^{*}\right)}}-e^{-\frac{(\lambda-\varepsilon)(1+2 \delta) \log x}{\Psi^{\prime}\left(\alpha^{*}\right)}} \\
& \geq(1-\varepsilon) e^{-\frac{(\lambda+\varepsilon)(1+\delta) \log x}{\Psi^{\prime}\left(\alpha^{*}\right)}}
\end{aligned}
$$


since $(\lambda+\varepsilon)(1+\delta)<(\lambda-\varepsilon)(1+2 \delta)$, and this implies that there exists $\delta \leq \zeta \leq 2 \delta$ such that $n_{x}=\left\lceil(1+\zeta)(\log x) / \Psi^{\prime}\left(\alpha^{*}\right)\right\rceil$ satisfies

$$
\mathbb{P}\left[N=n_{x}\right] \geq \frac{(1-\varepsilon) \Psi^{\prime}\left(\alpha^{*}\right) e^{-(\lambda+\varepsilon)(1+\delta) \log x / \Psi^{\prime}\left(\alpha^{*}\right)}}{\delta \log x} .
$$

Therefore, using (48) and denoting $\log J_{i}$ by $X_{i}$, we obtain

$$
\begin{aligned}
\mathbb{P}\left[\Pi_{N}^{0}>x\right] & \geq \mathbb{P}\left[N=n_{x}\right] \mathbb{P}\left[\sum_{i=1}^{n_{x}} \log J_{i}>\log x\right] \\
& \geq \frac{(1-\varepsilon) \Psi^{\prime}\left(\alpha^{*}\right) e^{-(\lambda+\varepsilon)(1+\delta) \log x / \Psi^{\prime}\left(\alpha^{*}\right)}}{\delta \log x} \mathbb{P}\left[\sum_{i=1}^{n_{x}} X_{i}>\frac{\Psi^{\prime}\left(\alpha^{*}\right)}{1+\delta} n_{x}\right] .
\end{aligned}
$$

Next, we perform an exponential change of measure for the probability on the right-hand side of (49). Let $\mathbb{P}_{n}^{*}$ be the probability measure on $\mathbb{R}^{n}$ defined by the probability measure $\mathbb{P}$ of the stationary and ergodic process $\left\{X_{i}\right\}_{i \geq 1}$

$$
\mathbb{P}_{n}^{*}\left(d x_{1}, \cdots, d x_{n}\right)=e^{\alpha^{*} S_{n}-\Psi_{n}\left(\alpha^{*}\right)} \mathbb{P}\left(d x_{1}, \cdots, d x_{n}\right),
$$

where $S_{n}=\sum_{i=1}^{n} X_{i}$ and $\Psi_{n}(\alpha) \triangleq \log \mathbb{E}\left[e^{\alpha S_{n}}\right]$ satisfying $n^{-1} \Psi_{n}(\alpha) \rightarrow \Psi(\alpha)$ in the neighborhood of $\alpha^{*}$. Thus,

$$
\begin{aligned}
\mathbb{P}\left[\sum_{i=1}^{n} X_{i}>\frac{\Psi^{\prime}\left(\alpha^{*}\right)}{1+\delta} n\right] & =\mathbb{E}_{n}^{*}\left[e^{-\alpha^{*} S_{n}+\Psi_{n}\left(\alpha^{*}\right)} \mathbf{1}\left(S_{n}>\frac{\Psi^{\prime}\left(\alpha^{*}\right)}{1+\delta} n\right)\right] \\
& \geq \mathbb{E}_{n}^{*}\left[e^{-\alpha^{*} S_{n}+\Psi_{n}\left(\alpha^{*}\right)} \mathbf{1}\left(\left|\frac{S_{n}}{n}-\Psi^{\prime}\left(\alpha^{*}\right)\right|<\frac{\Psi^{\prime}\left(\alpha^{*}\right) \delta}{1+\delta}\right)\right] \\
& \geq e^{-\alpha^{*} \frac{(1+2 \delta) \Psi^{\prime}\left(\alpha^{*}\right)}{1+\delta} n+\Psi_{n}\left(\alpha^{*}\right)} \mathbb{P}_{n}^{*}\left[\left|\frac{S_{n}}{n}-\Psi^{\prime}\left(\alpha^{*}\right)\right|<\frac{\Psi^{\prime}\left(\alpha^{*}\right) \delta}{1+\delta}\right] .
\end{aligned}
$$

Then, by Claim 1 on page 17 of [14], we know that

$$
\mathbb{P}_{n}^{*}\left[\left|\frac{S_{n}}{n}-\Psi^{\prime}\left(\alpha^{*}\right)\right|<\frac{\Psi^{\prime}\left(\alpha^{*}\right) \delta}{1+\delta}\right] \rightarrow 1 \text { as } x \rightarrow \infty,
$$

which, using (49) and setting $n=n_{x}$ in (50), yields

$$
\varliminf_{x \rightarrow \infty} \frac{\log \mathbb{P}\left[\Pi_{N}^{0}>x\right]}{\log x} \geq-\frac{(\lambda+\varepsilon)(1+\delta)}{\Psi^{\prime}\left(\alpha^{*}\right)}-\frac{\alpha^{*}(1+2 \delta)^{2}}{1+\delta}+\frac{(1+\delta) \Psi\left(\alpha^{*}\right)}{\Psi^{\prime}\left(\alpha^{*}\right)} .
$$

Finally, by passing $\varepsilon, \delta \rightarrow 0$ in the preceding equality and noting $\Psi\left(\alpha^{*}\right)=\lambda$, we prove the lower bound.

Proof of Theorem 11. We give a constructive proof based on the connection (duality) between the $M / G I / 1$ queue and the geometrically stopped multiplicative process.

Consider a $M / G I / 1$ queue with the service distribution $\mathbb{P}[S \geq t]=\bar{G}(t), t \geq 0$ and Poisson arrivals of rate $\lambda=\rho / \mathbb{E}[S], \mathbb{E}[S]<\infty$. Then, by the Pollaczeck-Khinchine formula (see, e.g., Theorem 5.7 on p. 237 of [5]), the stationary workload $Q$ of this $M / G I / 1$ queue is equal in distribution to $\sum_{i=1}^{N} H_{i}$, where $N,\left\{H_{i}\right\}_{i \geq 1}$ are independent with $\mathbb{P}[N>n]=\rho^{n}, n \geq 0$ and

$$
\mathbb{P}\left[H_{i} \leq x\right]=\frac{\int_{0}^{x} \mathbb{P}[S \geq s] d s}{\mathbb{E}[S]}=\frac{\int_{0}^{x} \bar{G}(s) d s}{\int_{0}^{\infty} \bar{G}(s) d s}=\mathbb{P}\left[\log J_{i} \leq x\right], \quad x \geq 0,
$$


where the last equality follows from the assumption. Now, using the preceding observation we show that there exists a RMP such that $M=e^{Q}$ satisfies

$$
\mathbb{P}[M>x]=\mathbb{P}[Q>\log x]=\mathbb{P}\left[\sum_{i=1}^{N} H_{i}>\log x\right]=\mathbb{P}\left[\sum_{i=1}^{N} \log J_{i}>\log x\right]=\mathbb{P}\left[\Pi_{N}^{0}>x\right],
$$

which proves the first claim of the theorem.

Next, using the additional assumptions of the theorem and applying Cramér-Lundberg theory for the $M / G I / 1$ queue (e.g., see Theorem 5.2 in Chapter XIII of [5]), we obtain

$$
\lim _{x \rightarrow \infty} \mathbb{P}[M>x] x^{\alpha^{*}}=\frac{(1-\rho) \int_{0}^{\infty} \bar{G}(y) d y}{\alpha^{*} \rho \int_{0}^{\infty} y e^{\alpha^{*} y} \bar{G}(y) d y},
$$

which, by (151), completes the proof.

Proof of Theorem 12. The second equality is implied by Theorem 10, and we only need to prove the first one. We begin with proving the upper bound. Recalling the definition of $\mathcal{B}_{n}^{l, \varepsilon}$ in Lemma $\left[5\right.$ and, for $n \geq 1,0<\varepsilon, \xi<1$, choosing $x^{\xi}>z_{0}>Z_{0}$, we obtain

$$
\begin{aligned}
\mathbb{P}\left[Z_{n}^{Z_{0}}>x\right] & \leq \mathbb{P}\left[Z_{n}^{\left\lfloor x^{\xi}\right\rfloor}>x\right] \\
& \leq \mathbb{P}\left[Z_{n}^{\left\lfloor x^{\xi}\right\rfloor}>x, \bigcap_{i=0}^{n-1}\left(\mathcal{B}_{i}^{\left\lfloor x^{\xi}\right\rfloor, \varepsilon}\right)^{C}\right]+\mathbb{P}\left[\bigcup_{i=0}^{n-1} \mathcal{B}_{i}^{\left\lfloor x^{\xi}\right\rfloor, \varepsilon}\right] \\
& \leq \mathbb{P}\left[\Pi_{n}^{0}(1+\varepsilon)^{n}>x^{1-\xi}\right]+n \mathbb{P}\left[\mathcal{B}_{0}^{\left\lfloor x^{\xi}\right\rfloor, \varepsilon}\right]
\end{aligned}
$$

which, by the independence of $N$ and $\left\{B_{n}^{i}(j), J_{n}\right\}$, implies

$$
\mathbb{P}\left[Z_{N}>x\right] \leq \mathbb{P}\left[\Pi_{N}^{0}(1+\varepsilon)^{N}>x^{1-\xi}\right]+\mathbb{E}[N] \mathbb{P}\left[\mathcal{B}_{0}^{\left\lfloor x^{\xi}\right\rfloor, \varepsilon}\right] .
$$

Next, define a new process $\left\{\Pi_{n}^{\varepsilon}=\Pi_{n}^{0}(1+\varepsilon)^{n}\right\}$. It is easy to see that, for $\varepsilon$ small enough, the sequence $\left\{\Pi_{n}^{\varepsilon}\right\}$ satisfies $n^{-1} \log \mathbb{E}\left[\left(\Pi_{n}^{\varepsilon}\right)^{\alpha}\right] \rightarrow \Psi(\alpha)+\alpha \log (1+\varepsilon)$. Therefore, by Theorem 10, we obtain

$$
\lim _{x \rightarrow \infty} \frac{\log \mathbb{P}\left[\Pi_{N}^{0}(1+\varepsilon)^{N}>x^{1-\xi}\right]}{\log x}=-(1-\xi) \alpha_{\varepsilon}^{*}
$$

where $\alpha_{\varepsilon}^{*}$ satisfies $\Psi\left(\alpha_{\varepsilon}^{*}\right)+\alpha_{\varepsilon}^{*} \log (1+\varepsilon)=0$. Combining (52), (53) and Lemma 6, we obtain

$$
\varlimsup_{x \rightarrow \infty} \frac{\log \mathbb{P}\left[Z_{N}>x\right]}{\log x} \leq-(1-\xi) \alpha_{\varepsilon}^{*}
$$

which, by passing $\varepsilon, \xi \rightarrow 0$, completes the proof of the upper bound.

Now, we prove the lower bound. Let $\left\{Z_{n, j}^{1}\right\}$ be i.i.d. copies of $\left\{Z_{n}^{1}\right\}$ given the common modulating process $\left\{J_{n}\right\}$. Then, noting that $Z_{n}^{y} \stackrel{d}{=} \sum_{j=1}^{y} Z_{n, j}^{1}$ for integer $y$ and using the union bound, we derive, for $0<\xi<1, n \geq 0$,

$$
\mathbb{P}\left[Z_{n}>x\right] \geq \frac{\left\lfloor x^{\xi}\right\rfloor}{x^{\xi}} \mathbb{P}\left[Z_{n}^{1}>x\right] \geq \frac{1}{x^{\xi}} \mathbb{P}\left[Z_{n}^{\left\lfloor x^{\xi}\right\rfloor}>x\left\lfloor x^{\xi}\right\rfloor\right] .
$$

Hence, recalling the definition of $\mathcal{C}_{n}^{l, \varepsilon}$ in Lemma 10, we obtain

$$
\begin{aligned}
\mathbb{P}\left[Z_{n}>x\right] & \geq \frac{1}{x^{\xi}} \mathbb{P}\left[Z_{n}^{\left\lfloor x^{\xi}\right\rfloor}>x\left\lfloor x^{\xi}\right\rfloor, \bigcap_{i=0}^{n-1}\left(\mathcal{C}_{i}^{\left\lfloor x^{\xi}\right\rfloor, \xi}\right)^{C}\right] \\
& \geq \frac{1}{x^{\xi}}\left(\mathbb{P}\left[\Pi_{n}^{0}(1-\xi)^{n}>x\right]-n \mathbb{P}\left[\mathcal{C}_{0}^{\left\lfloor x^{\xi}\right\rfloor, \xi}\right]\right),
\end{aligned}
$$


which, by the independence of $N$ and $\left\{B_{n}^{i}(j), J_{n}\right\}$, yields

$$
\mathbb{P}\left[Z_{N}>x\right] \geq \frac{1}{x^{\xi}}\left(\mathbb{P}\left[\Pi_{N}^{0}(1-\xi)^{N}>x\right]-\mathbb{E}[N] \mathbb{P}\left[\mathcal{C}_{0}^{\left\lfloor x^{\xi}\right\rfloor, \xi}\right]\right) .
$$

Then, by using the same approach as in the proof of the upper bound and Lemma 10, we can easily show that

$$
\varliminf_{x \rightarrow \infty} \frac{\log \mathbb{P}\left[Z_{N}>x\right]}{\log x} \geq-\alpha^{*} .
$$

Finally, by combining the upper bound and the lower bound, we finish the proof.

\subsection{Proof of Theorem 13}

Proof. We begin with the upper bound. Notice that when the system reaches stationarity, $N_{n}$ follows the Poisson distribution, and therefore, there exists $H>0$ such that

$$
\mathbb{P}\left[N_{0}>\lfloor H \log x\rfloor\right]=o\left(\frac{1}{x^{\alpha^{*}}}\right) .
$$

Denoting by $\left\{\Lambda_{i}\right\}_{i \geq 1}$ the i.i.d. copies of the random variable $\Lambda$ defined in Lemma 2 , we obtain

$$
\begin{aligned}
\mathbb{P}\left[Z_{s}>x\right] & \leq \mathbb{P}\left[\sum_{i=1}^{N_{0}} \Lambda_{i}>x\right] \\
& \leq \mathbb{P}\left[\sum_{i=1}^{\lfloor H \log x\rfloor} \Lambda_{i}>x\right]+\mathbb{P}\left[N_{0}>\lfloor H \log x\rfloor\right] \\
& \leq H \log x \mathbb{P}\left[\Lambda>\frac{x}{H \log x}\right]+\mathbb{P}\left[N_{0}>\lfloor H \log x\rfloor\right],
\end{aligned}
$$

which, in conjunction with Theorem 3 and equation (54), yields

$$
\varlimsup_{x \rightarrow \infty} \frac{\log \mathbb{P}\left[Z_{s}>x\right]}{\log x} \leq-\alpha^{*} .
$$

Next, we proceed with the lower bound. Construct a new process that has the same arrivals $\left\{A_{n}\right\}$ as described before but only allows at most one object to exist in the system. The construction goes as follows: all the new arrivals will be dropped if there is an object present in the system; similarly, when newly generated objects arrive to the empty system, only one object will be accepted while others will be dropped; the object, if any, evolves according to an i.i.d. copy of the modulated branching process $Z_{P}$. Denote the total size of the object in the new system at time $n$ by $\underline{Z}_{n}$, and observe that $\underline{Z}_{n}$ forms a renewal process. Then, taking out all the empty (idle) periods of the new system and concatenating the remaining periods sequentially yields a process equal in distribution to a reflected modulated branching process $\left\{\Lambda_{n}\right\}$, as defined in (2). Therefore, when the new system is in stationarity, we obtain, by the independence of $\left\{A_{n}\right\}$ and $Z_{P}$,

$$
\mathbb{P}\left[Z_{s}>x\right] \geq \mathbb{P}\left[\underline{Z}_{0}>x\right]=\mathbb{P}\left[\underline{Z}_{0}>0\right] \mathbb{P}[\Lambda>x],
$$

which, by Theorem 3, yields

$$
\varliminf_{x \rightarrow \infty} \frac{\log \mathbb{P}\left[Z_{s}>x\right]}{\log x} \geq-\alpha^{*} .
$$

Finally, combining (55) and (56) finishes the proof. 


\section{Acknowledgements}

We are thankful to the anonymous reviewers for their helpful comments and, in particular, we are grateful to a reviewer who pointed to us reference [32] that led to Theorem 7 .

\section{References}

[1] L. A. Adamic and B. A. Huberman. Zipf's law and the Internet. Glottometrics, 3:143-150, 2002.

[2] A. P. Allen, B. Li, and E. L. Charnov. Population fluctuations, power laws and mixtures of lognormal distributions. Ecology Letters, 4:1-3, 2001.

[3] L. A. N. Amaral, S. V. Buldyrev, S. Havlin, H. Leschhorn, P. Maass, M. A. Salinger, H. E. Stanley, and M. H. Stanley. Scaling behavior in economics: Empirical results for company growth. Journal de Physique I, 7:621-633, 1997.

[4] O. Arrhenius. Species and area. Journal of Ecology, 9(1):95-99, 1921.

[5] S. Asmussen. Applied Probability and Queues. Springer-Verlag, second edition, 1987.

[6] S. Asmussen, P. Fiorini, L. Lipsky, T. Rolski, and R. Sheahan. Asymptotic behavior of total times for jobs that must start over if a failure occurs. June 2007, preprint.

[7] K. Athreya and A. N. Vidyashankar. Branching processes. Springer-Verlag, 1972.

[8] F. Auerbach. Das gesetz der belvolkerungskoncentration. Petermanns Geographische Mitteilungen, 59:74-76, 1913.

[9] A. L. Barabási, R. Albert, and H. Jeong. Mean-field theory for scale-free random networks. Physica A, 272:173-187, 1999.

[10] P. Billingsley. Probability and Measure. John Wiley \& Sons, third edition, 1995.

[11] A. Blank and S. Solomon. Power laws in cities population, financial markets and Internet sites (scaling in systems with a variable number of components). Physica A, 287:279-288, 2000.

[12] L. Breslau, P. Cao, L. Fan, G. Phillips, and S. Shenker. Web caching and Zipf-like distributions: Evidence and implications. In Proceedings of IEEE INFOCOM 1999, volume 1, pages 126-134, New York, NY, USA, March 1999.

[13] J. Brujic, R. I. H. Z., K. A. Walther, and J. M. Fernandez. Single-molecule force spectroscopy reveals signatures of glassy dynamics in the energy landscape of ubiquitin. Nature Physics, 2(4):282$286,2006$.

[14] J. A. Bucklew. Large deviation techniques in decision, simulation, and estimation. John Wiley \& Sons, 1990.

[15] J. M. Carlson and J. Doyle. Highly optimized tolerance: A mechanism for power laws in designed systems. Physics Review E, 60(2):1412-1427, 1999.

[16] D. Champernowne. A model of income distribution. Economic Journal, 63:318-351, 1953.

[17] C.-S. Chang. Stability, queue length, and delay of deterministic and stochastic queueing networks. IEEE Transactions on Automatic Control, 39(5):913-931, May 1994.

[18] E. F. Connor and E. D. McCoy. The statistics and biology of the species-area relationship. The American Naturalist, 113(6):791-833, June 1979.

[19] M. Crovella and A. Bestavros. Self-similarity in World Wide Web traffic: Evidence and possible causes. IEEE/ACM Transactions on Networking, 5(6):835-846, December 1997.

[20] C. Cunha, A. Bestavros, and M. Crovella. Characteristics of World Wide Web client-based traces. Technical Report TR-95-010, Department of Computer Science, Boston University, 1995. 
[21] C. Dagum. A systematic approach to the generation of income distribution models. Journal of Income Distribution, 61:105-126, 1996.

[22] L. de Haan, S. I. Resnick, H. Rootzén, and C. G. de Vries. Extremal behaviour of solutions to a stochastic difference equation with applications to ARCH processes. Stochastic Processes and Their Applications, 32:213-224, 1989.

[23] B. de Saporta. Tail of the stationnary solution of the stochastic equation $y_{n+1}=a_{n} y_{n}+b_{n}$ with markovian coefficients. Stochastic Processes and their Applications, 115(12):1954-1978, December 2005 .

[24] A. Dembo and O. Zeitouni. Large Deviations Techniques and Applications. Springer-Verlag, second edition, 1998.

[25] A. B. Downey. The structural cause of file size distributions. In Proceedings of ACM SIGMETRICS 2001, pages 328-329, New York, NY, USA, 2001.

[26] M. Faloutsos, P. Faloutsos, and C. Faloutsos. On power-law relationships of the Internet topology. In Proceedings of the ACM SIGCOMM 1999 Conference, pages 251-262, 1999.

[27] P. M. Fiorini, R. Sheahan, and L. Lipsky. On unreliable computing systems when heavy-tails appear as a result of the recovery procedure. In MAMA 2005 Workshop, Banff, AB, Canada, June 2005; ACM SIGMETRICS Performance Evaluation Review, Volume 33, Issue 2, pp. 15-17, September 2005.

[28] X. Gabaix. Zipf's law for cities: an explanation. The Quarterly Journal of Economics, 114(3):739$767,1999$.

[29] X. Gabaix, P. Gopikrishnan, V. Plerou, and H. E. Stanley. A theory of power-law distributions in financial market fluctuations. Nature, 423:267-270, 15 May 2003.

[30] A. Ganesh, N. O'Connell, and D. Wischik. Big Queues. Springer-Verlag, 2004.

[31] P. W. Glynn and W. Whitt. Logarithmic asymptotics for steady-state tail probabilities in a singleserver queue. Studies in Applied Probability, 31:131-156, 1994.

[32] C. M. Goldie. Implicite renewal theory and tails of solutions of random equations. The Annals of Applied Probability, 1(1):126-166, Feburary 1991.

[33] W.-B. Gong, Y. Liu, V. Misra, and D. Towsley. Self-similarity and long range dependence on the Internet: a second look at the evidence, origins and implications. Computer Networks, 48:377-399, June 2005.

[34] B. A. Huberman and L. A. Adamic. Evolutionary dynamics of the World Wide Web. Technical report, Xerox Palo Alto Research Center, 1999. Appears as a brief communication in Nature, 399, p. 130, 1999.

[35] B. A. Huberman and L. A. Adamic. The nature of markets in the World Wide Web. Quarterly Journal of Economic Commerce 1, pages 5-12, 2000.

[36] M. A. Huynen and E. van Nimwegen. The frequency distribution of gene family sizes in complete genomes. Molecular Biology and Evolution, 15(5):583-9, May 1998.

[37] Y. M. Ioannides and H. G. Overman. Zipf's law for cities : an empirical examination. Regional Science and Urban Economics, 33(2):127-137, 2003.

[38] P. R. Jelenković and A. Lazar. On the dependence of the queue tail distribution on multiple time scales of ATM multiplexers. In Proceedings of the Conference on Information Sciences and Systems, pages 435-440, Baltimore, MD, March 1995.

[39] P. R. Jelenković, A. A. Lazar, and N. Semret. The effect of multiple time scales and subexponentiality in MPEG video streams on queueing behavior. IEEE Journal of Selected Areas in Communications, 15(6):1052-1071, 1997. 
[40] P. R. Jelenković and P. Momčilović. Asymptotic loss probabilityin a finite buffer fluid queue with heterogeneous heavy-tailed on-off processes. Annals of Applied Probability, 13(2), 2003.

[41] P. R. Jelenković and J. Tan. Is ALOHA causing power law delays? In Proeedings of the 20th International Teletraffic Congress, Ottawa, Canada, June 2007; Lecture Notes in Computer Science, No 4516, pp. 1149-1160, Springer-Verlag, 2007. (Best Student Paper Award).

[42] P. R. Jelenković and J. Tan. Modulated branching processes and origins of power laws. In Proceedings of the Forty-Fourth Annual Allerton Conference on Communication, Control, and Computing, Allerton House, UIUC, Illinois, USA, September 2006.

[43] P. R. Jelenković and J. Tan. Are end-to-end acknowlegements causing power law delays in large multi-hop networks? In the 14th Informs Applied Probability Conference, Eindhoven, July 9-11 2007.

[44] P. R. Jelenković and J. Tan. Can retransmissions of superexponential documents cause subexponential delays? In Proceedings of IEEE INFOCOM 200\%, pages 892-900, Anchorage, Alaska, USA, (submitted August 2006, accepted November 2006), May 2007.

[45] P. R. Jelenković and J. Tan. Characterizing heavy-tailed distributions induced by retransmissions. Technical Report EE2007-09-07, Department of Electrical Engineering, Columbia University, New York, NY, September 2007. Eprint arXiv:0709.1138v2.

[46] J. E. Keeley. Relating species abundance distributions to species-area curves in two mediterraneantype shrublands. Diversity and Distributions, 9:253-259, 2003.

[47] H. Kesten. Random difference equations and renewal theory for products of random matrices. Acta Mathematica, 131:207-248, 1973.

[48] R. Kumar, P. Raghavan, S. Rajagopalan, D. Sivakumar, A. Tomkins, and E.Upfal. Stochastic models for the web graph. In Proceedings of the 41st Annual Symposium on Foudations of Computer Science, pages 57-65, 2000.

[49] W. E. Leland, W. Willinger, M. S. Taqqu, and D. V. Wilson. On the self-similar nature of Ethernet traffic. ACM SIGCOMM Computer Communication Review, 25(1):202-213, 1995.

[50] M. Levy and S. Solomon. Dynamical explanation for the emergence of power law in a stock market model. International Journal of Modern Physics C, 7:65-72, 1996.

[51] M. Levy and S. Solomon. Power laws are logarithmic boltzmann laws. International Journal of Modern Physics C, 7:595-601, 1996.

[52] M. Levy and S. Solomon. Spontaneous scaling emergence in generic stochastic systems. International Journal of Modern Physics C, 7(5):745-756, 1996.

[53] R. M. Loynes. The stability of a queue with non-independent inter-arrival and service times. Proceedings of the Cambridge Philosophical Society, 58:497-520, 1962.

[54] N. M. Luscombe, J. Qian, Z. Zhang, T. Johnson, and M. Gerstein. The dominance of the population by a selected few: power-law behaviour applies to a wide variety of genomic properties. Genome Biology, 3(8):research0040.0041-0040.0047, 2002.

[55] B. Mandelbrot. The Pareto-Lev́y law and the distribution of income. International Economic Review, 1:79-106, 1960.

[56] A. Medina, I. Matta, and J. Byers. On the origin of power laws in Internet topologies. Computer Communication Review, 30(2):18-28, 2000.

[57] M. Mitzenmacher. A brief history of generative models for power law and lognormal distributions. Internet Mathematics, 1(2):226-251, 2004.

[58] M. Mitzenmacher. Dynamic models for file sizes and double Pareto distributions. Internet Mathematics, 1(3):305 - 333, 2004. 
[59] M. Olvera-Cravioto, J. Blanchet, and P. W. Glynn. On the transition from heavy traffic to heavy tails for the $\mathrm{M} / \mathrm{G} / 1$ queue: the regularly varying case. private communication, 2006.

[60] V. Pareto. Cours d'Economie Politique, Droz, Geneva, Switzerland, 1896.

[61] J. Parr. A note on the size distribution of cities over time. Journal of Urban Economics, VIII, pages 199-212, 1985.

[62] J. B. Plotkin, M. D. Potts, D. W. Yu, S. Bunyavejchewin, R. Condit, R. Foster, S. Hubbell, J. LaFrankie, N. Manokaran, H.-S. Lee, R. Sukumar, M. A. Nowak, and P. S. Ashton. Predicting species diversity in tropical forests. Proceedings of the National Academy of Sciences of the United States of America, 97(20):10850-10854, September 26, 2000.

[63] F. Preston. The canonical distribution of commonness and rarity. Ecology, 43(2):185-215,410-432, 1962.

[64] W. J. Reed. The Pareto, Zipf and other power laws. Economics Letters, 74:15-19, 2001.

[65] W. J. Reed. The Pareto law of incomes - an explanation and an extension. Physica A, 319:469-486, 2003.

[66] W. J. Reed and B. D. Hughes. From gene families and genera to incomes and internet file sizes: Why power laws are so common in nature. Physical Review E, 66(6):067103, December 2002.

[67] W. J. Reed and M. Jorgensen. The double Pareto - lognormal distribution - a new parametric model for size distributions. Communications in Statistics: Theory and Methods, 33(8):1733 $1753,2004$.

[68] K. Rosen and M. Resnick. The size distribution of cities: An examination of the Pareto law and primacy. Journal of Urban Economics, VIII, pages 165-186, 1980.

[69] A. Rzhetsky and S. M. Gomez. Birth of scale-free molecular networks and the number of distinct DNA and protein domains per genome. Bioinformatics, 17:988-996, 2001.

[70] R. Sheahan, L. Lipsky, P. M. Fiorini, and S. Asmussen. On the completion time distribution for tasks that must restart from the beginning if a failure occurs. In MAMA 2006 Workshop, Saint-Malo, France, June 2006; ACM SIGMETRICS Performance Evaluation Review, Volume 34, Issue 3, pp. 24-26, December 2006.

[71] D. Sornette and R. Cont. Convergent multiplicative processes repelled from zero: power laws and truncated power laws. Journal de Physique I, 7(3):431-444, March 1997.

[72] S. Wichmann. On the power-law distribution of language family sizes. Journal of Linguistics, 41(1):117-131, March 2005.

[73] G. Zipf. Human Behavior and the Principle of Least Effort. Addison-Wesley, Cambridge, MA, 1949. 